(C) 2000 International Press

Adv. Theor. Math. Phys. 6 (2000) 1835-1891

\title{
Uncovering Infinite
}

\section{Symmetries on $[\mathrm{p}, \mathrm{q}]$}

7-branes: Kac-Moody

\section{Algebras and Beyond}

\author{
Oliver DeWolfe, Tamás Hauer, Amer Iqbal, \\ and Barton Zwiebach
}

\author{
Center for Theoretical Physics \\ Laboratory for Nuclear Science \\ Department of Physics \\ Massachusetts Institute of Technology \\ Cambridge, MA 02139, USA \\ odewolfe,hauer,iqbal@ctp.mit.edu \\ zwiebach@irene.mit.edu
}

\begin{abstract}
In a previous paper we explored how conjugacy classes of the modular group classify the symmetry algebras that arise on type IIB [p,q] 7-branes. The Kodaira list of finite Lie algebras completely fills the elliptic classes as well as some parabolic classes.
\end{abstract}

e-print archive: http://xxx.lanl.gov/hep-th/9812209 
Loop algebras of $E_{N}$ fill additional parabolic classes, and exotic finite algebras, hyperbolic extensions of $E_{N}$ and more general indefinite Lie algebras fill the hyperbolic classes. Since they correspond to brane configurations that cannot be made into strict singularities, these non-Kodaira algebras are spectrum generating and organize towers of massive BPS states into representations. The smallest brane configuration with unit monodromy gives rise to the loop algebra $\widehat{E}_{9}$ which plays a central role in the theory. We elucidate the patterns of enhancement relating $E_{8}, E_{9}, \widehat{E}_{9}$ and $E_{10}$. We examine configurations of 24 7-branes relevant to type IIB compactifications on a two-sphere, or Ftheory on K3. A particularly symmetric configuration separates the 7-branes into two groups of twelve branes and the massive BPS spectrum is organized by $E_{10} \oplus E_{10}$.

\section{Introduction}

One of the most intriguing aspects of string theory is that the entire massive spectrum appears to be associated with the spontaneous breaking of a large and rather mysterious symmetry. This symmetry is not restored in any familiar vacuum, but it clearly reflects the underlying structure of the theory. Unbroken symmetries, while no doubt important, are essentially associated to the massless spectrum, and represent only a small part of the complete structure. One of our objectives in this paper is to explain how the simple compactification of F-theory on an elliptic K3, equivalent to type IIB superstrings on a two-sphere with 24 7-branes [1], provides a natural setting where infinite symmetries associated to the massive BPS spectrum of the theory can be identified explicitly. These infinite symmetries are completely natural generalizations of the finite exceptional symmetries $E_{6}, E_{7}$ and $E_{8}$ that are by now well understood in the 7-brane setup $[2,3,4,5]$. Indeed, the main difference lies in that the finite algebras arise from brane configurations where the branes can be brought together, while this is not the case for the infinite algebras. Instead the infinite set of BPS strings and BPS string junctions stretching between the branes always represent massive states and essentially correspond to the generators of the infinite dimensional algebra. 
This is another example of how interesting configurations of type IIB $[\mathrm{p}, \mathrm{q}]$ 7-branes are not limited to those where the branes can be brought together. In a previous paper [6], entire families of configurations realizing finite $A D E$ algebras were explored, extending the $D_{N}, E_{N}$ and Argyres-Douglas $H_{N}$ series beyond those cases that exist in the Kodaira classification of K3 singularities. D3-brane probes in the background of such non-collapsible configurations realize physically relevant $4 \mathrm{D}$ theories with eight supercharges such as $S U(2)$ Seiberg-Witten theory with $N_{f}=0,1,2,3$ flavors, and some of the theories with $E_{N}$ global symmetry which also arise in toroidal compactifications of the $6 \mathrm{D}$ tensionless string theory.

Although only finite Lie algebras are associated to singularities in the Kodaira classification, in configurations that cannot collapse many types of algebras arise. As was found in [7], affine exceptional algebras appear whenever the intersection form of string junctions realized on a brane configuration reproduces the affine Cartan matrix. These brane configurations are no more exotic than the ones realizing their finite counterparts. Some new features appear in these cases, including an imaginary root junction corresponding to a genus one curve in $\mathrm{K} 3$, and a relationship between the asymptotic $(p, q)$ charge of a junction and a Lie algebraic property, the level $k$ of the associated representation. More exotic infinite algebras also appear. We find, for example, hyperbolic exceptional algebras, many Lorentzian Kac-Moody algebras, as well as loop extensions of infinite algebras and other algebras that are not even of Kac-Moody type.

In [6], it was seen how 7-brane configurations realizing the various finite Lie algebras were classified by the total monodromy around the branes, along with the additional data of the number of branes and the possible asymptotic charges on supported junctions. Since the monodromy is only unique up to global $S L(2, \mathbf{Z})$ transformations, this classification determines a correspondence between 7-brane Lie algebras and conjugacy classes of $S L(2, \mathbf{Z})$. These classes are well-studied in the mathematical literature, and are divided by their $S L(2, \mathbf{Z})$-invariant trace into the elliptic, parabolic and hyperbolic classes. The finite algebras spanned the elliptic classes, and fell into some of the parabolic classes and hyperbolic classes of negative trace as well.

A powerful mathematical correspondence exists between conjugacy 
classes of $S L(2, \mathbf{Z})$ and equivalence classes of binary quadratic forms. In [6], it was seen how this correspondence determines the asymptotic charge quadratic form $f(p, q)$, introduced in [5] and used in [8] to find the global symmetry representations of various $(p, q)$ dyons in the Seiberg-Witten and $E_{N}$ theories. In addition, it was found that $f(p, q)$ determines how one algebra enhances to another when an additional $[p, q] 7$-brane is added: when $f(p, q)<-1$ only a new $u(1)$ factor appears, $f(p, q)=-1$ enhances $\mathcal{G}$ to $\mathcal{G} \oplus A_{1}$, and $-1<f(p, q)<1$ indicates the enhancement to another finite algebra.

The various infinite-dimensional algebras arising on 7-brane configurations fit into the classification by $S L(2, \mathbf{Z})$ conjugacy classes, filling many of the parabolic and hyperbolic classes of positive trace. These algebras are shown to appear when one enhances a finite algebra with a $[p, q] 7$-brane satisfying $f(p, q) \geq 1$. Affine algebras appear for $f(p, q)=1$, while other Kac-Moody algebras with indefinite Cartan matrix appear when $f(p, q)>1$. All these Kac-Moody algebras appear when their Cartan matrices are realized in the junction intersection form.

Not all of the infinite-dimensional algebras arising on 7-branes are Kac-Moody, however. A central example which we study in some detail is the loop algebra $\widehat{E}_{9}$, which is a loop enhancement of the affine algebra $E_{9}$, the same way that the affine algebras can be viewed as loop algebras of their finite counterparts. The algebra $\widehat{E}_{9}$ has no Cartan matrix and so cannot be Kac-Moody. The associated configuration, which we call $\widehat{\mathbf{E}}_{\mathbf{9}}$, has unit monodromy; its twelve branes are in fact the fewest that can realize such a monodromy. It follows that factors of $\widehat{\mathbf{E}}_{\mathbf{9}}$ are invisible to the total monodromy of some configuration. There are enhancements of $\widehat{E}_{9}$ as well, all of which are not Kac-Moody, such as the loop algebras of the $E_{N}$ series for $N>9$. The configuration we are associating to $\widehat{E}_{9}$ was first studied in [9], where it was argued that the collapse of these branes corresponds to a decompactification limit.

The classification, summarized in Table 5, has a rich and interesting structure. The collapsible brane configurations corresponding to Kodaira singularities occupy the elliptic classes and some of the parabolic classes. Configurations associated to hyperbolic classes of negative trace realize various exotic finite algebras. The affine exceptional algebras appear in certain parabolic classes, as do the non-Kac- 
Moody loop algebras such as $\widehat{E}_{9}$. The hyperbolic classes of positive trace are filled with various other infinite-dimensional algebras, including hyperbolic algebras like $E_{10}$. Laid on top of everything is a kind of $\widehat{E}_{9}$ periodicity, as a given conjugacy class will also realize configurations with arbitrary numbers of unit-monodromy $\widehat{\mathbf{E}}_{\mathbf{9}}$ configurations appended.

We also begin to examine the question of which of these algebras can arise on a physical compactification of F-Theory on K3. Just as the finite $D_{N}$ and $E_{N}$ algebras organize the BPS spectra of the field theories mentioned above, the algebra of the entire brane configuration on K3 should organize the BPS states of Type IIB string compactifications. We show how global considerations project out certain junctions looping around all the 7-branes on the sphere, thus reducing the rank of the algebra appearing. A particularly interesting configuration with the 24 branes split in two groups of twelve branes makes manifest an $E_{10} \oplus E_{10}$ subalgebra. This splitting is very special in that there is a one to one correspondence between the root lattice of $E_{10} \oplus E_{10}$ and the K3 homology captured by the junction lattice.

The application of various infinite-dimensional Lie algebras to string theory is an interesting subject studied by many people. Generalized Kac-Moody algebras have been developed by Borcherds [10] based on vertex algebras of toroidal compactification of bosonic strings. These algebras, particularly $E_{10}[11]$, were also investigated by Gebert and Nicolai [12]. Moore and Harvey [13, 14] studied the algebras of BPS states, and in particular they identified a role for $E_{10}$ in the context of threshold corrections for $\mathcal{N}=2 D=4$ heterotic compactifications [13]. Generalized Kac-Moody algebras also arise in the context of fivebranes [15]. Gritsenko and Nikulin [16] developed and studied a variant of the theory of Lorentzian Kac-Moody algebras involving hyperbolic generalized Cartan matrices.

Our analysis, however, differs from many of the above in that it does not involve vertex algebras; the relevant Lie algebras are realized from intersection of cycles in K3. The use of configurations giving infinitedimensional algebras to the calculation of the spectrum of probe D3brane theories is still an open problem which we intend to address in the future.

In section 2, we review a few important properties of 7-branes, string 
junctions and the associated algebras. Affine algebras on 7-branes are discussed in section 3 , as well as their intersection form and the imaginary root junction. In section 4 we introduce the loop algebra $\vec{E}_{9}$ and its associated brane configuration. Infinite-dimensional algebras of indefinite type are studied in section 5 , these include the exceptional hyperbolic series. In section 6 we synthesize this study into a classification of all these configurations according to their $S L(2, \mathbf{Z})$ conjugacy class, as presented in Table 5. Section 7 discusses the application of these results to two manifolds admitting elliptic fibration, $\mathcal{B}_{9}$ and $\mathrm{K} 3$. Finally, the mathematical techniques used to calculate the multiplicities of $S L(2, \mathbf{Z})$ conjugacy classes are detailed in the appendix.

\section{Review of 7-branes and Lie Algebras}

We shall introduce the infinite-dimensional algebras arising on 7-brane configurations by exploring how they arise as enhancements of more familiar, finite algebras. Let us first review the 7-brane configurations realizing finite algebras, and their supported junctions.

Consider a configuration $\mathbf{G}=\mathbf{X}_{\mathbf{z}_{1}} \ldots \mathbf{X}_{\mathbf{z}_{n}}$ of 7-branes $\mathbf{X}_{\mathbf{z}_{\mathbf{i}}}$, where we use a charge-vector notation $\mathbf{z}_{i}=\left[p_{i}, q_{i}\right]$ to label the charges of each brane. Each 7-brane has an associated monodromy

$$
K_{\mathbf{z}}=\mathbb{1}+\mathbf{z z}^{T} S=\left(\begin{array}{cc}
1+p q & -p^{2} \\
q^{2} & 1-p q
\end{array}\right)
$$

and the total monodromy $K$ is the product of the individual monodromy matrices. The charges of the 7-branes are only invariant up to global $S L(2, \mathbf{Z})$ transformations $g$, under which $K_{\mathbf{z}} \rightarrow g K_{\mathbf{z}} g^{-1}$, and the relocation of branch cuts. Moving the branch cut of the brane $\mathbf{X}_{\mathbf{z}_{\mathbf{2}}}$ through $\mathbf{X}_{\mathbf{z}_{\mathbf{1}}}$ exchanges their order in the canonical presentation and modifies the charges $\mathbf{z}_{1}$, as

$$
\begin{aligned}
\mathbf{X}_{\mathbf{z}_{1}} \mathbf{X}_{\mathbf{z}_{2}} & =\mathbf{X}_{\mathbf{z}_{2}} \mathbf{X}_{\left(\mathbf{z}_{1}+\left(\mathbf{z}_{1} \times \mathbf{z}_{2}\right) \mathbf{z}_{2}\right)}, \\
\text { where } \quad \mathbf{z}_{1} \times \mathbf{z}_{2} & \equiv-\mathbf{z}_{1}^{T} S \mathbf{z}_{2}=\operatorname{det}\left(\begin{array}{cc}
p_{1} & p_{2} \\
q_{1} & q_{2}
\end{array}\right)
\end{aligned}
$$

The physics should not depend on either of these transformations, and configurations will be considered equivalent if they can be mapped into 
one another by global $S L(2, \mathbf{Z})$ and moving branch cuts [4]. The total monodromy changes as $K \rightarrow g K g^{-1}$ under global $S L(2, \mathbf{Z})$, and is invariant under relocating branch cuts.

Consider an algebra $\mathcal{G}$ of rank $r$ realized on $\mathrm{G}$. The objects charged under $\mathcal{G}$ are string junctions $\mathbf{J}$, which are webs of $(p, q)$ strings with prongs ending on the 7-branes. A string junction carries the charge of a 7-brane if it has a prong on the brane and/or it crosses the brane's branch cut. These two possibilities, combined in a way that is invariant under a Hanany-Witten transformation, define the invariant charge $Q \mathbf{x}_{i}(\mathbf{J})$ of the junction $\mathbf{J}$ associated to the 7 -brane $\mathbf{X}_{i}$. The set of invariant charges completely determines the junction's algebraic properties. The $Q \mathbf{x}_{i}$ are integers, and thus the space of junctions is a lattice. Instead of the $Q_{\mathbf{x}_{i}}$, a junction on $\mathbf{G}$ can be characterized by a $\mathcal{G}$ weight vector $\lambda$, as well as asymptotic charges $(p, q)$. The self-intersection of a junction is its norm on the lattice of junctions, and is determined by the length-squared of its Lie algebra weight vector $\lambda \cdot \lambda$ and a binary quadratic form in the asymptotic charges, $f_{K}(p, q)[5]$ :

$$
(\mathbf{J}, \mathbf{J})=-\lambda \cdot \lambda+f_{K}(p, q) .
$$

It was shown in [6] that the asymptotic charge quadratic form $f_{K}(p, q)$ associated to $\mathbf{G}$ is determined simply by the monodromy ${ }^{1}$,

$$
f_{K}(\mathbf{z})=\frac{1}{2-t} \mathbf{z}^{T} S K \mathbf{z} \equiv \frac{Q_{K}(\mathbf{z})}{2-t}
$$

where $S=\left(\begin{array}{cc}0 & -1 \\ 1 & 0\end{array}\right)$ is the usual $S L(2, \mathbf{Z})$ generator and $t \equiv \operatorname{Tr} K$.

Although the $S L(2, \mathbf{Z})$ conjugacy class of $K$ carries most of the information about the algebra, it is not always sufficient for the classification of the configurations. One has to specify the number of 7-branes, and possible constraints on the $(p, q)$ asymptotic charges the branes can support. The latter can be characterized in the following way [6]. For the charges $\mathbf{z}_{i}$ of all 7 -branes in $\mathbf{G}$, define

$$
\ell \equiv \begin{cases}\operatorname{gcd}\left\{\mathbf{z}_{i} \times \mathbf{z}_{j}, \text { for all } i, j\right\} & \text { for mutually nonlocal branes } \\ 0 & \text { for mutually local branes. }\end{cases}
$$

\footnotetext{
${ }^{1}$ The correspondence between conjugacy classes of $S L(2, \mathbf{Z})$ and equivalence classes of binary quadratic forms was discussed in another context in [17].
} 
It is manifest that $\ell$ is invariant under global $S L(2, \mathbf{Z})$ transformations, and it can be shown it also does not change when branch cuts are relocated. The asymptotic charges $\mathbf{z}=(p, q)$ are constrained to obey

$$
\mathbf{z} \times \mathbf{z}_{i}=0 \quad(\bmod \ell),
$$

where $\mathbf{z}_{i}$ denotes the charges of some 7 -brane in $\mathbf{G}$. The constraint (2.7) will be the same regardless of the choice of $\mathbf{z}_{i}$ thanks to (2.6).

In the next few sections, we shall begin consider a finite configuration $\mathbf{G}$ with algebra $\mathcal{G}$ and proceed to add a single new brane $\mathbf{Z}$ characterized by charges $\mathbf{z}=[p, q]$, exploring the infinite algebras $\mathcal{G}^{\text {enh }}$ that can appear on the total configuration $\mathrm{G}^{e n h}=\mathrm{GZ}$. This procedure was considered for finite enhancement in [6] (sec.5), where it was shown that on the total configuration $\mathbf{G}^{e n h}$, a generic junction with zero asymptotic charges can be expressed as

$$
\mathbf{J}=-n \mathbf{z}+n\left(p \boldsymbol{\omega}^{p}+q \boldsymbol{\omega}^{q}\right)+\sum_{i} a_{i} \boldsymbol{\omega}^{i},
$$

and can thus be specified by its $\mathcal{G}$ weight and the invariant charge $-n$ on the $\mathbf{Z}$ brane. Each such junction $\mathbf{J}=(n, \lambda)$ is identified with a root of $\mathcal{G}^{\text {enh }}$. The self-intersection $\mathbf{J}^{2}$ of these junctions satisfies the relation,

$$
\lambda \cdot \lambda=-\mathbf{J}^{2}+n^{2}\left(f_{K}(\mathbf{z})-1\right),
$$

where $f_{K}(\mathbf{z})$ is the value of the charge quadratic form of $\mathbf{G}$ evaluated on the charges of $\mathbf{Z}$. Since $\lambda \cdot \lambda \geq 0$ and the BPS condition requires $\mathbf{J}^{2} \geq-2$ [8], equation (2.9) need not have solutions for all "grades" $n$. Indeed, for the finite cases considered in [6], there are only solutions at finitely many $n$. For $f(\mathbf{z})<-1$ the only solution is at $n=0$, meaning that the only roots are the original ones of $\mathcal{G}$, and $\mathcal{G}^{e n h}=\mathcal{G} \oplus u(1)$. This $u(1)$ arises since the space of junctions without asymptotic charges increases its dimension by one. For $f(\mathbf{z})=-1$ precisely two new roots $( \pm 1, \lambda=0)$ appear, giving $\mathcal{G}^{\text {enh }}=\mathcal{G} \oplus A_{1}$ (unless $n= \pm 1$ are incompatible with $(2.8))$. For values $-1<f(\mathbf{z})<1$ there is a solution for finitely many nonzero grades, and $\mathcal{G}^{\text {enh }}$ is some finite algebra of rank $r+1$.

As we shall see, when $f(\mathbf{z}) \geq 1$ we find that $\mathbf{G}^{\text {enh }}$ realizes some infinite-dimensional Lie algebra. In most cases the algebra $\mathcal{G}^{\text {enh }}$ can be identified by finding a set of simple roots. The simple roots then determine the Cartan matrix and Dynkin diagram of $\mathcal{G}^{e n h}$. Simple roots 
of Kac-Moody Lie algebras are always real, meaning the corresponding junctions have $\mathbf{J}^{2}=-2$. In most cases a single new simple root is necessary, and as explained in [6], this enhanced simple root will be $\boldsymbol{\alpha}_{0}=\left(n_{0}, \theta_{<}\right)$where the grade $n_{0}$ is the lowest positive grade satisfying (2.9), and $\theta_{<}$is the lowest weight of the Weyl orbit at this grade. In a few exotic cases two additional (linearly dependent) simple roots will be necessary to produce a basis.

The results we shall find for how $f(\mathbf{z})$ controls the kind of enhanced algebra $\mathcal{G}^{\text {enh }}$ are summarized in Table 1 . The case $\mathbf{G}=\mathbf{A}_{\mathbf{N}}$, where the configuration is composed of $N+1$ mutually local branes realizing $\mathcal{G}=A_{N}$ is exceptional, adding a mutually local brane enhances to $\mathbf{A}_{\mathbf{N}+\mathbf{1}}$, but any mutually nonlocal brane does not enhance $\mathcal{G}$ at all, but instead allows both asymptotic charges to be realized on junctions.

\begin{tabular}{|c|c|}
\hline$f(\mathbf{z})$ & $\mathcal{G}^{\text {enh }}$ \\
\hline$f(\mathbf{z})<-1$ & $\mathcal{G} \oplus u(1)$ \\
$f(\mathbf{z})=-1$ & $\mathcal{G} \oplus A_{1}$ \\
$-1<f(\mathbf{z})<1$ & finite \\
$f(\mathbf{z})=1$ & affine or loop \\
$f(\mathbf{z})>1$ & indefinite \\
\hline
\end{tabular}

Table 1: Algebraic enhancements of brane configurations. Adding one brane to an existing configuration $\mathbf{G}$ realizing a finite algebra $\mathcal{G}$, the asymptotic charge form $f(\mathbf{z})$ of $\mathbf{G}$ evaluated on the new brane determines the type of $\mathcal{G}^{\text {enh }}$.

\section{Affine Enhancement: $f(\mathbf{z})=1$}

Consider the case when the charge quadratic form associated to a finite algebra $\mathcal{G}$ can take the value $f(\mathbf{z})=1$ for some charge $\mathbf{z}$. Add a brane with that charge and examine the resulting enhancement. In this case equation (2.9) for the $\mathcal{G}$ weight vector of the new roots reduces to

$$
\lambda \cdot \lambda=-\mathbf{J}^{2},
$$

where the dependence on the grade $n$ drops out. Hence, unlike the case for $f(\mathbf{z})<1$, this equation can be satisfied for all $n$. There are 
solutions with $\mathbf{J}^{2}=-2$ as long as the $\mathcal{G}$ weight vector $\lambda$ is a root, and so there is an infinite tower of root vectors, with the roots of $\mathcal{G}$ repeated at each grade $n$. (There is an important exception when $\mathcal{G}$ has, in addition to roots, other weight vectors satisfying $\lambda \cdot \lambda=2$. In this case the enhanced algebra is still affine, but it is not $\widehat{\mathcal{G}}$. We discuss this in section 3.2.) Moreover, we have solutions satisfying $\mathbf{J}^{2}=0$ with $\lambda=0$, corresponding to an imaginary root junction, as we describe in the next subsection. This is precisely the structure of the affine Lie algebra $\widehat{\mathcal{G}}$. The realization of these algebras on 7-branes was first studied in [7]. Here we review those results, and simplify and clarify a few issues. For background on infinite-dimensional Lie algebras, see [18].

\subsection{The imaginary root junction $\delta$}

Due to the degeneracy of the affine Cartan matrix, affine algebras possess an "imaginary" root $\delta=\alpha_{0}+\sum_{i=1}^{r} c^{i} \alpha_{i}$, where $r$ is the rank of the associated finite algebra and $c^{i}$ are the marks of that algebra. It has zero intersection with all roots:

$$
\delta \cdot \alpha_{i}=0, \quad i=0 \ldots r,
$$

and as a consequence it also obeys $\delta \cdot \delta=0$. All vectors of the form $n \delta$ for $n \in \mathbf{Z}$ are roots of the affine algebra, with degeneracy equal to $r$. For finite algebras, all root junctions satisfy $(\boldsymbol{\alpha}, \boldsymbol{\alpha})=-2$; in contrast, for the affine case we expect to see an imaginary root junction $\delta$ satisfying $(\boldsymbol{\delta}, \boldsymbol{\delta})=0$. Because of (3.2) we also must have $\left(\boldsymbol{\delta}, \boldsymbol{\alpha}_{i}\right)=0$ for all $\boldsymbol{\alpha}_{i}$, and therefore the imaginary root has $\lambda=0$. It is identified as the solution of (3.1) with $\mathbf{J}^{2}=\lambda \cdot \lambda=0$, and $n=1$.

For the affine configurations constructed in [7], it was noted that $\boldsymbol{\delta}$ could always be presented as a string looping around the brane configuration. We now explain why this is guaranteed by the algebraic properties of the monodromy matrices. Consider the total monodromy $K_{\mathbf{Z}} K$ obtained by adding to a brane configuration with monodromy $K$ a $\mathbf{Z}$ brane of charge $\mathbf{z}$ satisfying $f_{K}(\mathbf{z})=1$. We claim that this total monodromy admits an eigenvector $\mathbf{z}_{\mathbf{0}}=\left(p_{0}, q_{0}\right)$ with eigenvalue one:

$$
K_{\mathbf{Z}} K \mathbf{z}_{\mathbf{0}}=\mathbf{z}_{\mathbf{0}} .
$$

Consider a loop of string with charge $\mathbf{z}_{\mathbf{0}}=\left(p_{0}, q_{0}\right)$ that first crosses the branch cut of the $\mathcal{G}$ configuration, becoming $K \mathbf{z}_{\mathbf{0}}$, and then crosses 
the cut of $\mathbf{Z}$, becoming $\mathbf{z}_{\mathbf{0}}$ again. Charge conservation requires that the charge acquired from the $\mathcal{G}$ configuration is all lost again on the $\mathbf{Z}$ brane, and hence $K \mathbf{z}_{\mathbf{0}}-\mathbf{z}_{\mathbf{0}}=n \mathbf{z}$ for some $n$. Using $(K-\mathbb{1})^{-1}=$ $\left(K^{-1}-\mathbb{1}\right) /(2-t)$, we can now calculate $\mathbf{z}_{\mathbf{0}}$ in terms of $\mathbf{z}$, finding

$$
\mathbf{z}_{\mathbf{0}}=\frac{n}{2-t}\left(K^{-1}-\mathbb{1}\right) \mathbf{z}
$$

where $t=\operatorname{Tr} K$ and $n$ can be chosen to be the minimum integer that permits integer values for $\mathbf{z}_{\mathbf{0}}$. We must now verify that (3.3) holds. Substituting (3.4) and recalling the expression for the monodromy of a single brane (2.1), (3.3) is equivalent to

$$
\left(\mathbb{1}+\mathbf{z z}^{T} S\right)(\mathbb{1}-K) \mathbf{z}=\left(K^{-1}-\mathbb{1}\right) \mathbf{z} .
$$

Using $K^{-1}=t \mathbb{1}-K$ and $\mathbf{z}^{T} S \mathbf{z}=0$, we finally obtain the condition

$$
\left(\mathbf{z}^{T} S K \mathbf{z}\right) \mathbf{z}=(2-t) \mathbf{z}
$$

which by $(2.5)$ holds precisely when $f_{K}(\mathbf{z})=1$. This completes the proof: the junction $\delta$ is a loop with charge $\mathbf{z}_{\mathbf{0}}$ as shown in Fig. 1 .

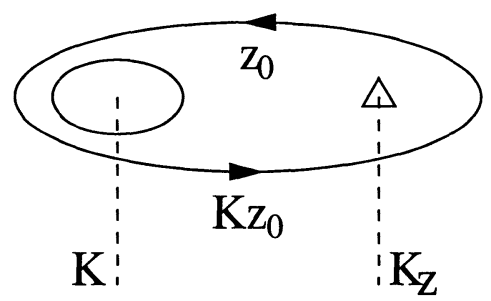

Figure 1: The imaginary root junction as a loop surrounding the enhanced configuration.

Because it traces out a simple Jordan closed curve around the branes, the junction clearly has $\delta^{2}=0$. In addition, it can be chosen not to intersect any simple root, all of which are localized on the brane configuration. Therefore all intersection numbers with roots vanish and the associated weight vector vanishes. Thus the presentation of $\boldsymbol{\delta}$ as a loop makes its key properties manifest. 


\begin{tabular}{|c|c|c|c|}
\hline $\mathcal{G}$ & Brane Configuration & $K$ & $f_{K}(p, q)$ \\
\hline \multirow{3}{*}{$A_{N}$} & $\mathbf{A}_{\mathbf{N}}=\mathbf{A}^{N+1}$ & $\left(\begin{array}{cc}1 & -N-1 \\
0 & 1\end{array}\right)$ & $-\frac{1}{N+1} p^{2}$ \\
\hline & $\mathbf{H}_{\mathbf{N}}=\mathbf{A}^{N+1} \mathbf{C}$ & $\left(\begin{array}{cc}2 & -3-2 N \\
1 & -N-1\end{array}\right)$ & $\begin{array}{c}\frac{1}{N+1}\left\{-p^{2}+(N+3) p q\right. \\
\left.-(3+2 N) q^{2}\right\}\end{array}$ \\
\hline & $\tilde{\mathbf{H}}_{\mathbf{N}}=\mathbf{A}^{N} \mathbf{X}_{[\mathbf{0},-1]} \mathbf{C}$ & $\left(\begin{array}{cc}1 & -N-1 \\
1 & -N\end{array}\right)$ & $\begin{array}{c}\frac{1}{N+1}\left\{-p^{2}+(N+1) p q\right. \\
\left.-(N+1) q^{2}\right\}\end{array}$ \\
\hline$D_{N}$ & $\mathbf{D}_{\mathbf{N}}=\mathbf{A}^{N} \mathbf{B C}$ & $\left(\begin{array}{cc}-1 & N-4 \\
0 & -1\end{array}\right)$ & $\frac{N-4}{4} q^{2}$ \\
\hline \multirow[t]{2}{*}{$E_{N}$} & $\mathbf{E}_{\mathbf{N}}=\mathbf{A}^{N-1} \mathbf{B C C}$ & $\left(\begin{array}{cc}-2 & 2 N-9 \\
-1 & N-5\end{array}\right)$ & $\begin{array}{c}\frac{1}{9-N}\left\{p^{2}+(3-N) p q\right. \\
\left.+(2 N-9) q^{2}\right\}\end{array}$ \\
\hline & $\tilde{\mathbf{E}}_{\mathbf{N}}=\mathbf{A}^{N} \mathbf{X}_{[2,-1]} \mathbf{C}$ & $\left(\begin{array}{cc}-3 & 3 N-11 \\
-1 & N-4\end{array}\right)$ & $\begin{array}{l}\frac{1}{9-N}\left\{p^{2}+(1-N) p q\right. \\
\left.\quad+(3 N-11) q^{2}\right\}\end{array}$ \\
\hline
\end{tabular}

Table 2: Brane configurations, monodromies and the charge quadratic form for $A_{N}, D_{N}$ and $E_{N}$ algebras. The two series $\left(\mathbf{H}_{\mathbf{N}}\right.$ and $\left.\tilde{\mathbf{H}}_{\mathbf{N}}\right)$ realizing the $A_{N}$ algebras are equivalent. The $\mathbf{A}_{N}$ series of mutually local branes supports only asymptotic $p$-charge. The two series $\left(\mathbf{E}_{\mathbf{N}}\right.$ and $\tilde{\mathbf{E}}_{\mathbf{N}}$ ) realizing $E_{N}$ are equivalent for $N \geq 2$.

\subsection{Configurations realizing affine algebras}

Finite $A D E$ algebras can be realized on 7-branes, as reviewed in [6]. In this section we explore which of the affinizations of these are realized on branes. In Table 2, we summarize several useful series of brane configurations realizing finite algebras, and the monodromies and asymptotic charge forms associated to these configurations. $\mathbf{H}_{\mathbf{N}}$ and $\tilde{\mathbf{H}}_{\mathbf{N}}$ are equivalent for all $N$, while $\mathbf{E}_{\mathbf{N}}$ and $\tilde{\mathbf{E}}_{\mathbf{N}}$ are equivalent only for $N \geq 2$. A $[p, q]$ 7-brane is denoted $\mathbf{X}_{[\mathbf{p}, \mathbf{q}]}$, while certain useful 7-branes are abbreviated $\mathbf{A} \equiv \mathbf{X}_{[\mathbf{1}, \mathbf{0}]}, \mathbf{B} \equiv \mathbf{X}_{[\mathbf{1},-1]}$, and $\mathbf{C} \equiv \mathbf{X}_{[\mathbf{1}, \mathbf{1}]}$.

Affine exceptional algebras. Only exceptional affine algebras $\widehat{E}_{N}$ seem to appear on 7-branes, while generic $\widehat{A}_{N}$ and $\widehat{D}_{N}$ are absent. 
To any $\mathbf{E}_{\mathbf{N}}$ configuration we can add a brane with $\mathbf{z}=[3,1]$, and since $f(3,1)=1 \forall N$ we obtain affine enhancement (see Table 2). Equivalent configurations are obtained by adding a brane with charges $g \mathbf{z}$ with $g$ in the centralizer of the monodromy, $g K_{E_{N}} g^{-1}=K_{E_{N}}$, as $f_{K}(g \mathbf{z})=f_{K}(\mathbf{z})$. We thus define the series

$$
\widehat{\mathbf{E}}_{\mathbf{N}} \equiv \mathbf{E}_{\mathbf{N}} \mathbf{X}_{[3,1]}=\mathbf{A}^{N-1} \mathbf{B C C X}_{[3,1]}=\mathbf{A}^{N-1} \mathbf{B C B C} .
$$

The monodromy is readily calculated to be

$$
K\left(\widehat{E}_{N}\right)=\left(\begin{array}{cc}
1 & 9-N \\
0 & 1
\end{array}\right) .
$$

The $\delta$ junction is a $(-1,0)$ string going counterclockwise, and as expected $(-1,0)$ is an eigenvector of the monodromy with eigenvalue plus one. The invariant charges of $\boldsymbol{\delta}$ are

$$
\delta=\mathbf{b}+\mathbf{c}_{1}+\mathbf{c}_{2}-\mathbf{x}_{[3,1]}=\mathbf{b}_{1}+\mathbf{c}_{1}-\mathbf{b}_{2}-\mathbf{c}_{2},
$$

and so from the point of view of enhancing $\mathbf{E}_{\mathbf{N}}$ it has $n=1$ (see (2.8)). Note $\boldsymbol{\delta}$ has no support on the A-branes.

Notice that $\operatorname{Tr} K\left(\widehat{\mathbf{E}}_{\mathbf{N}}\right)=2$. This must be the case for any $S L(2, \mathbf{Z})$ matrix having a unit eigenvalue. Configurations of mutually local branes also have a monodromy with this property, but the string that winds around has the same charge as the branes, and is therefore equivalent to the trivial zero junction.

The $\tilde{\mathbf{E}}_{\mathbf{N}}$ configurations may be enhanced as well. Adding a $[4,1]$ brane gives $f_{K}(4,1)=1$ (see Table 2 ) resulting in

$$
\widehat{\tilde{\mathbf{E}}}_{\mathrm{N}}=\mathbf{A}^{N} \mathbf{X}_{[2,-1]} \mathbf{C X}[4,1]
$$

and in fact $K\left(\widehat{\mathbf{E}}_{\mathbf{N}}\right)=K\left(\widehat{\tilde{\mathbf{E}}}_{\mathbf{N}}\right)$. The imaginary root $\boldsymbol{\delta}$ is still a $(-1,0)$ loop, and has invariant charges

$$
\boldsymbol{\delta}=\mathbf{x}_{[2,-1]}+2 \mathbf{c}-\mathbf{x}_{[4,1]} .
$$

$\widehat{\mathbf{E}}_{\mathbf{N}}$ and $\widehat{\tilde{\mathbf{E}}}_{\mathbf{N}}$ are equivalent for $N \geq 2$, since the $S L(2, \mathbf{Z})$ conjugation by $K_{A}$ required to demonstrate the equivalence of the finite configurations turns the $[4,1]$ brane into a $[3,1]$ brane (see equation (2.11) in [6].) We shall use the $\widehat{\mathbf{E}}_{\mathbf{N}}$ presentation for simplicity. 
For $N=0$, however, there is only $\tilde{\mathbf{E}}_{\mathbf{0}}$ and thus we must consider $\widehat{\tilde{\mathbf{E}}}_{\mathbf{0}}=\mathbf{X}_{[\mathbf{2},-\mathbf{1}]} \mathbf{C X}_{[\mathbf{4}, \mathbf{1}]}$. The finite configuration $\tilde{\mathbf{E}}_{\mathbf{0}}$ has no algebra, and all supported junctions have nonzero $(p, q)$. In the affine case, all junctions with vanishing asymptotic charge are proportional to the imaginary root $\boldsymbol{\delta}$.

For $N=1$ we have $\mathbf{E}_{\mathbf{1}} \not \tilde{\mathbf{E}}_{\mathbf{1}}$, and their affinizations are also inequivalent. The case of $\widehat{\mathbf{E}}_{\mathbf{1}}=\mathbf{B C B C}$ was studied in detail in [7]. It was seen to give the algebra $\widehat{E}_{1}=\widehat{A_{1}}=\widehat{s u(2)}$. On the other hand the configuration

$$
\widehat{\tilde{\mathbf{E}}}_{1}=\operatorname{AX}_{[2,-1]} \mathrm{CX}_{[4,1]}=\mathrm{BAX}_{[1,-2]} \mathrm{C},
$$

does not support any zero asymptotic charge junctions with $\mathbf{J}^{2}=-2$, but has a $\widehat{u(1)}$ algebra instead. The configuration $\widehat{\tilde{\mathbf{E}}}_{\mathbf{1}}$ supports all possible asymptotic charges as $\tilde{\mathbf{E}}_{\mathbf{1}}$ does, while for $\widehat{\mathbf{E}}_{\mathbf{1}}$, like $\mathbf{E}_{\mathbf{1}}$, only charges obeying $p+q=0(\bmod 2)$ are permitted.

When a single $\mathbf{A}$-brane is added, both $\widehat{\mathbf{E}}_{\mathbf{1}}$ and $\widehat{\tilde{\mathbf{E}}}_{\mathbf{1}}$ become equivalent to $\widehat{\mathbf{E}}_{\mathbf{2}}$. The algebra $E_{2}=A_{1} \oplus u(1)$ is not semisimple, and $E_{3}=A_{2} \oplus A_{1}$ is semisimple but not simple. The associated affine brane configurations realize the algebras $\widehat{E}_{2}$ and $\widehat{E}_{3}$, defined as the quotients of $\widehat{A}_{1} \oplus \widehat{u(1)}$ and $\widehat{A}_{2} \oplus \widehat{A}_{1}$, respectively, by central elements. We will discuss this further in section 3.4. The configurations $\widehat{\mathbf{E}}_{\mathrm{N}}, N=4,5,6,7,8$ realize the algebras $\widehat{A}_{4}, \widehat{D}_{5}, \widehat{E}_{6}, \widehat{E}_{7}$ and $\widehat{E}_{8}$. These configurations and their algebras are summarized in Table 3 .

\begin{tabular}{|c|c|c|c|c|c|c|c|c|c|}
\hline$\widehat{\mathbf{E}}_{\mathbf{0}}$ & $\widehat{\mathbf{E}}_{\mathbf{1}}$ & $\widehat{\tilde{\mathbf{E}}}_{\mathbf{1}}$ & $\widehat{\mathbf{E}}_{\mathbf{2}}$ & $\widehat{\mathbf{E}}_{\mathbf{3}}$ & $\widehat{\mathbf{E}}_{\mathbf{4}}$ & $\widehat{\mathbf{E}}_{\mathbf{5}}$ & $\widehat{\mathbf{E}}_{\mathbf{6}}$ & $\widehat{\mathbf{E}}_{\mathbf{7}}$ & $\widehat{\mathbf{E}}_{\mathbf{8}}$ \\
\hline$\{\widehat{0}\}$ & $\widehat{A}_{1}$ & $\widehat{u(1)}$ & $\widehat{A_{1}} \oplus \widehat{u(1)} / \sim$ & $\widehat{A}_{2} \oplus \widehat{A}_{1} / \sim$ & $\widehat{A}_{4}$ & $\widehat{D}_{5}$ & $\widehat{E}_{6}$ & $\widehat{E}_{7}$ & $\widehat{E}_{8}$ \\
\hline
\end{tabular}

Table 3: The inequivalent affine exceptional configurations for $N \leq 8$ and their algebras.

Finally, the $\widehat{\mathbf{E}}_{N}$ configurations with $N \geq 9$ realize algebras $\widehat{E}_{N}$ which are identified as loop algebras of $E_{N}$. Since $E_{N}$ with $N \geq 9$ is not a finite algebra, the loop extensions are not Kac-Moody algebras, and do not have Dynkin diagrams or Cartan matrices. The simplest case of $\widehat{\mathbf{E}}_{\mathbf{9}}$ is also the smallest nontrivial configuration with unit monodromy, and will be discussed in some detail in section 4 . 
The absence of $\widehat{A}_{N}$ and $\widehat{D}_{N}$ algebras. We have claimed that the $\widehat{A}_{N}$ and $\widehat{D}_{N}$ affine algebras do not appear on 7-brane configurations. Let us discuss attempting to affinize members of the $\mathbf{A}, \mathbf{H}$ and $\mathbf{D}$ series. Examining the quadratic forms in Table 2, we see the equation $f(\mathbf{z})=1$ has a solution only for the $\mathbf{H}_{\mathbf{4}}, \mathbf{H}_{\mathbf{7}}, \mathbf{H}_{\mathbf{8}}, \mathbf{D}_{\mathbf{5}}$ and $\mathbf{D}_{\mathbf{8}}$ configurations. Two of those are already familiar since $\mathbf{H}_{\mathbf{4}} \cong \mathbf{E}_{\mathbf{4}}$ and $\mathbf{D}_{\mathbf{5}} \cong \mathbf{E}_{\mathbf{5}}$. We claim that the others also enhance to affine exceptional algebras.

Take for example $\mathbf{D}_{\mathbf{8}}$, for which $f(p, q)=q^{2}$ (see Table 2). Here $\mathbf{D}_{\mathbf{8}} \mathbf{X}_{[\mathbf{p}, \mathbf{1}]}$ for any $p$ is an affine enhancement, since $f(p, 1)=1$. However the algebra is not $\widehat{D}_{8}$ but $\widehat{E_{8}}$. A quick way to see this is that $\mathbf{D}_{\mathbf{N}} \rightarrow$ $\mathbf{E}_{\mathbf{N}+\mathbf{1}}$ by adding a $[p, 1]$ brane, as discussed in section 6 of [6] and summarized in this paper in Table 4. (For $p=1$ this is manifest from the definitions of the $\mathbf{D}$ and $\mathbf{E}$ series). Therefore $\mathbf{D}_{\mathbf{8}} \rightarrow \mathbf{E}_{\mathbf{9}} \equiv \widehat{\mathbf{E}}_{\mathbf{8}}$. We obtain the larger adjoint 248 of $E_{8}$ because the $D_{8}$ weights with $\lambda \cdot \lambda=2$ include not just the adjoint 120, but also the spinors $\mathbf{1 2 8}, \overline{\mathbf{1 2 8}}$, and so additional root vectors appear upon enhancement. Choosing $p=0$ for concreteness, one can show using the conjugacy rules given in section 6 of [5] that the $\overline{\mathbf{1 2 8}}$ junctions cannot end on $\mathbf{X}_{[\mathbf{p}, \mathbf{1}]}$, while the $\mathbf{1 2 8}$ can end on it with $n$ odd and the $\mathbf{1 2 0}$ with $n$ even. The new simple root must have the lowest value of $|n|$, so we must take the lowest root of the spinor 128, which can have $n=1$. We then obtain the Cartan matrix of $\widehat{E}_{8}$, not $\widehat{D}_{8}$, which would have appeared had the simple root been in the 120. The combination of the $D_{8}$ adjoint 120 at grade $2 n$ and spinor $\mathbf{1 2 8}$ at grade $2 n+1$ for each $n$ gives a complete $E_{8}$ adjoint 248.

Similarly, we have $\mathbf{H}_{\mathbf{7}} \rightarrow \widehat{\mathbf{E}}_{\mathbf{7}}$ by adding a [5,1] brane, and $\mathbf{H}_{\mathbf{8}} \rightarrow \widehat{\mathbf{E}}_{\mathbf{8}}$ by adding a $[4,1]$ brane. The latter is in accord with the enhancement $\mathbf{H}_{\mathbf{N}}$ to $\mathbf{E}_{\mathbf{N}+\mathbf{1}}$ noted in Table 4 . In both cases the new simple root arises from additional weights of $\lambda \cdot \lambda=2$.

Equivalence of $\widehat{\mathbf{E}}_{\mathbf{8}}$ and $\mathbf{E}_{\mathbf{9}}$. Let us finally give an example showing how two different configurations realizing $\widehat{E}_{8}=E_{9}$ can be mapped into one another by global $S L(2, \mathbf{Z})$ transformations and relocating branch cuts. The configuration $\mathbf{E}_{\mathbf{8}}=\mathbf{A}^{7} \mathbf{B C C}$ can be enhanced both to $\widehat{\mathbf{E}}_{\mathbf{8}}=\mathbf{A}^{7} \mathbf{B C B C}$ and $\mathbf{E}_{\mathbf{9}}=\mathbf{A}^{8} \mathbf{B C C}$, and each realizes an $E_{9}$ algebra, as discussed in [7]. The $\mathbf{E}_{\mathbf{9}}$ configuration supports an imaginary root junction $\delta$ which is a $(3,1)$ string looping counterclockwise, with 
invariant charges

$$
\boldsymbol{\delta}=-\sum_{i=1}^{8} \mathbf{a}_{i}+4 \mathbf{b}+2 \mathbf{c}_{1}+2 \mathbf{c}_{2} .
$$

We shall now prove that these two configurations are indeed equivalent. We find that

$$
K\left(\widehat{\mathbf{E}}_{\mathbf{8}}\right)=T=\left(\begin{array}{ll}
1 & 1 \\
0 & 1
\end{array}\right) \stackrel{g}{\cong}\left(\begin{array}{ll}
-2 & 9 \\
-1 & 4
\end{array}\right)=-T^{2} S T^{-4}=K\left(\mathbf{E}_{\mathbf{9}}\right)
$$

where similarity is implemented by

$$
K\left(\widehat{\mathbf{E}}_{\mathbf{8}}\right)=g K\left(\mathbf{E}_{\mathbf{9}}\right) g^{-1}, \quad \text { with } \quad g=\left(\begin{array}{ll}
0 & -1 \\
1 & -3
\end{array}\right) .
$$

This monodromy turns $\mathbf{E}_{\mathbf{9}}$ into $\mathbf{E}_{\mathbf{9}}{ }^{\prime}=\left(\mathbf{X}_{[\mathbf{0 , 1}]}\right)^{8} \mathbf{X}_{[\mathbf{1}, \mathbf{4}]}\left(\mathbf{X}_{[\mathbf{1 , 2}]}\right)^{2}$, which can be shown to be equivalent to $\widehat{\mathbf{E}}_{\mathbf{8}}$ after relocating branch cuts:

$$
\begin{aligned}
& \left(\mathbf{X}_{[\mathbf{0}, \mathbf{1}]}\right)^{8} \mathbf{X}_{[\mathbf{1}, \mathbf{4}]}\left(\mathbf{X}_{[\mathbf{1}, \mathbf{2}]}\right)^{2} \\
& =\left(\mathbf{X}_{[0,1]}\right)^{8} \mathbf{X}_{[\mathbf{1 , 2}]} \mathbf{A ~ X}_{[\mathbf{1 , 2}]}=\left(\mathbf{X}_{[\mathbf{0 , 1}]}\right)^{7} \mathbf{C X}_{[\mathbf{0 , 1 ]}} \mathbf{A X}_{[\mathbf{1 , 2}]} \\
& =\mathrm{CA}^{7} \mathbf{X}_{[0,1]} \mathbf{A X}_{[1,2]}=\mathbf{C A}^{7} \mathbf{B C} \mathbf{X}_{[0,1]} \\
& =\mathrm{CA}^{3}\left(\mathrm{~A}^{4} \mathrm{BC}\right) \mathrm{X}_{[0,1]}=\mathrm{CA}^{3} \mathrm{X}_{[0,1]} \mathbf{A}^{4} \mathbf{B C} \\
& =\mathrm{CX}_{[3,1]} \mathrm{A}^{7} \mathrm{BC}=\mathrm{BCA}^{7} \mathrm{BC}=\mathrm{A}^{6} \mathrm{BX}_{[0,1]} \mathrm{CBC} \\
& =\mathrm{A}^{7} \mathrm{BCBC} \text {. }
\end{aligned}
$$

\subsection{The affine intersection form}

Here we discuss the junction intersection form for the $\widehat{\mathbf{E}}_{\mathbf{N}}$ configuration $(N \leq 8)$, continuing the discussion of [7]. ${ }^{2}$ We see how the junction selfintersection is related to the length of the corresponding affine weight vector. This formula involves the asymptotic charge form of the finite $\mathbf{E}_{\mathbf{N}}$ configuration.

The Cartan matrix of an affine algebra $\widehat{\mathcal{G}}$ is degenerate, and therefore it cannot be inverted. The inner product of two affine weight

\footnotetext{
${ }^{2}$ Some notation has been modified; the junction parameter there called $\tilde{n}$ is now called $n$, while the Lie algebraic quantity there denoted $n$ is now $\bar{n}$.
} 
vectors is not a straightforward generalization of the finite case. In fact the weight vector itself is specified not just by the Dynkin labels $a_{i}$, but by a grade $\bar{n}$ as well. Then inner product of the $\widehat{\mathcal{G}}$ weight vectors can be shown to be

$$
\left(\lambda_{1} \cdot \lambda_{2}\right)_{\widehat{\mathcal{G}}}=\left(\lambda_{1} \cdot \lambda_{2}\right)_{\mathcal{G}}+\bar{n}_{1} k_{2}+k_{1} \bar{n}_{2}
$$

where the level $k$ is a particular combination of the Dynkin labels defined by

$$
k \equiv(\lambda \cdot \delta)=a_{0}+\sum_{i=1}^{r} c^{i} a_{i},
$$

with $c^{i}$ the marks of $\mathcal{G}$, and $\left(\lambda_{1} \cdot \lambda_{2}\right)_{\mathcal{G}}$ the inner product of the $\mathcal{G}$ weight vectors defined by the $a_{i}, i=1 \ldots r$.

In our junction picture, we enhance an $E_{N}$ algebra to $\widehat{E}_{N}$ by adding a single brane. This adds only one new degree of freedom to the possible junctions appearing on the configuration, so it is not immediately obvious how the two new affine quantities, $\bar{n}$ and $a_{0}$ (or $\bar{n}$ or $k$ ) can be accounted for. What happens is that the Dynkin labels and asymptotic charges are not independent, but instead the level $k$ is a linear combination of $p$ and $q$. In the canonical realization of $\widehat{\mathbf{E}}_{\mathbf{N}}$,

$$
k=-q=-(\mathbf{J}, \boldsymbol{\delta}),
$$

where, as discussed before, $\boldsymbol{\delta}$ is a counterclockwise loop of charge $(-1,0)$ intersecting no root junction and carrying no asymptotic charge. As in the finite case, there are two parameters not specified by the Dynkin labels. They are not, however, $p$ and $q$, but rather $p$ and $n$, the latter being the coefficient of $\boldsymbol{\delta}$ in the junction, and coinciding with the grade $n$ found in enhancing $E_{N} \rightarrow \widehat{E}_{N}$. In a highest weight affine representation the Lie algebraic grade $\bar{n}$ is fixed conventionally to be zero for the highest weight vector. In the junction picture the natural grade of a junction is given by $n$. There can be an overall offset between $n$ and $\bar{n}$. For all weight vectors $\lambda$ in an affine representation, and all associated junctions $\mathbf{J}(\lambda)$ we set

$$
n(\mathbf{J})=\bar{n}(\lambda)+n_{0},
$$

where $n_{0}$ is a constant throughout the representation. Here $n_{0}$ can be identified as the junction grade of the highest weight vector. 


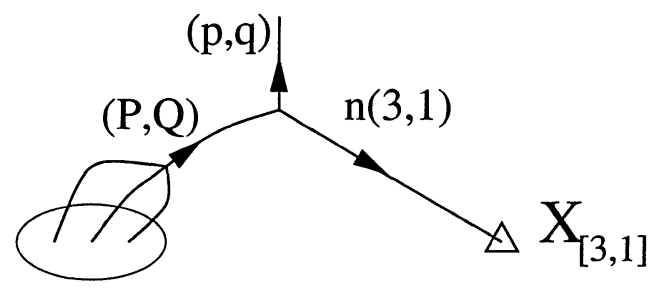

Figure 2: A junction on an affine configuration.

Let us now calculate the intersection form. As depicted in figure 2, an arbitrary junction on an $\widehat{\mathbf{E}}_{\mathbf{N}}$ affine configuration can be written

$$
\mathbf{J}=-n \mathbf{z}+P \boldsymbol{\omega}^{p}+Q \boldsymbol{\omega}^{q}+\sum_{i=1}^{r} a_{i} \boldsymbol{\omega}^{i},
$$

with $\mathbf{z}=[3,1]$ the charges of the enhancing brane. The total asymptotic charge of $\mathbf{J}$ is $(p, q)=-n(3,1)+(P, Q)$. The self-intersection is then

$$
\mathbf{J}^{2}=-n^{2}-(\lambda \cdot \lambda)_{E_{N}}+f_{E_{N}}(P, Q)+n \mathbf{z} \times\left(\begin{array}{c}
P \\
Q
\end{array}\right),
$$

where the first term is the contribution of the prongs on $\mathbf{X}_{[\mathbf{3}, \mathbf{1}]}$, the next two are the self-intersection of the $\mathbf{E}_{\mathbf{N}}$ junction with weight $\lambda$ and charges $(P, Q)$, and the last term is from the junction point. In order to express the result in terms of the true asymptotic charges $(p, q)$ we use

$$
f_{E_{N}}(P, Q)=f_{E_{N}}(p+3 n, q+n)=f_{E_{N}}(p, q)+n(p-q)+n^{2}
$$

which follows by explicit computation using the quadratic form listed in Table 2. One then arrives at

$$
\mathbf{J}^{2}=-(\lambda \cdot \lambda)_{E_{N}}-2 n k+f_{E_{N}}(p, q) .
$$

This is the intersection formula for $\widehat{\mathbf{E}}_{\mathbf{N}}$ junctions in terms of the length squared of the $E_{N}$ weight vector, the junction grade $n$ and the level $k$, and the asymptotic charge quadratic form of $\mathbf{E}_{\mathbf{N}}$. When $n=0$ we recover the intersection result for $\mathbf{E}_{\mathbf{N}}$. Since $(\lambda \cdot \lambda)_{\widehat{E}_{N}}=(\lambda \cdot \lambda)_{E_{N}}+2 \bar{n} k$, the first two terms in (3.24) are recognized as the affine inner product up to the shift (3.20) between $n$ and $\bar{n}$ :

$$
\mathbf{J}^{2}=-(\lambda \cdot \lambda)_{\widehat{E}_{N}}-2 n_{0} k+f_{E_{N}}(p, q),
$$


The last two terms in the right-hand side are constant over a given representation. Thus we see the affine intersection form does not have the neat partition into Lie algebraic and $(p, q)$ charge parts present in the finite cases, as in (2.4). Instead the two are mixed, thanks to the relation (3.19) between the level and the asymptotic charges.

The self-intersection of an affine junction naturally involves the charge quadratic form of the corresponding finite configuration, but one may wonder how the quadratic form defined from the affine monodromy $K\left(\widehat{\mathbf{E}}_{\mathbf{N}}\right)$ is related to (3.24). In the affine case we have $t=2$, and (2.5) cannot be used.. We must go back to the original definition of $f_{K}$ as the self-intersection of a singlet junction as discussed in section 3 of [6]. For such a singlet only $p$ and $n$ will be nonzero, since the vanishing of the Dynkin labels forces $q=0$ because of (3.19). We realize the singlet by a string with charges $\overline{\mathbf{z}}=(\bar{p}, \bar{q})$ that crosses the branch cut of the affine configuration and joins itself to form an asymptotic string z. We see $\mathbf{z}=((9-N) \bar{q}, 0)$. This junction then has intersection

$$
\mathbf{J}^{2}=\mathbf{z} \times \overline{\mathbf{z}}=(9-N) \bar{q}^{2}=\frac{1}{9-N} p^{2},
$$

and defines the affine quadratic form $f_{\widehat{E}_{N}}$. This is just what we expect from (3.24) for a junction with $a_{i}=k=0$, since $f_{E_{N}}(p, 0)=\frac{1}{9-N} p^{2}$. The fact that $n$ drops out of the intersection formula can be understood since an asymptotic string with $k=-q=0$ can wind around the configuration $n$ times without changing its asymptotic charge or its selfintersection. This result is similar to the asymptotic charge quadratic form for a set of mutually local branes $\mathbf{A}_{\mathbf{N}}$, where $f=\frac{1}{N+1} p^{2}$, which should not be surprising since the monodromies have the same structure: $K\left(\mathbf{A}_{\mathbf{N}}\right)=\left(\begin{array}{cc}1 & -(N+1) \\ 0 & 1\end{array}\right)$. We will have more to say about this relationship in section 6 .

\subsection{Affine semisimple algebras}

It is not immediately obvious what the affinizations of the non-simple algebras $E_{2}=A_{1} \oplus u(1)$ and $E_{3}=A_{2} \oplus A_{1}$ are, and the algebras appearing on the configurations $\widehat{\mathbf{E}}_{2}$ and $\widehat{\mathbf{E}}_{3}$ must be considered carefully. We examine $\widehat{\mathbf{E}}_{\mathbf{3}}$ for concreteness, but similar remarks apply to $\widehat{\mathbf{E}}_{\mathbf{2}}$.

Although one might think the affine enhancement of $E_{2}$ would be 
$\widehat{A_{2}} \oplus \widehat{A_{1}}$, this is incorrect. This algebra has two distinct imaginary roots, $\delta_{1}$ and $\delta_{2}$, and correspondingly weight vectors have two levels $k_{1}$ and $k_{2}$ and two grades $n_{1}$ and $n_{2}$. The $\widehat{\mathbf{E}}_{\mathbf{3}}$ configuration, however, supports only one $\boldsymbol{\delta}$ junction, and thus a single level $k$, as expected since the rank of the resulting algebra should exceed that of $E_{3}$ by one. Furthermore, neither $\widehat{A_{2}} \oplus A_{1}$ nor $A_{2} \oplus \widehat{A_{1}}$ is the correct algebra, though they have the correct rank. In these cases, only one of the simple pieces is affine and has roots at every grade; the other simple component would have roots only at $n=0$. Since the roots of both simple pieces can satisfy (2.9) at arbitrary grade, we expect to have the complete root system of $A_{2} \oplus A_{1}$ at every grade $n$.

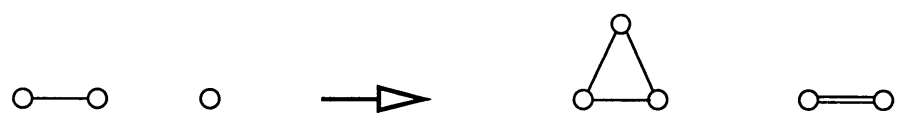

Figure 3: The enhancement of the Dynkin diagram of $E_{3}$ to that of $\widehat{E}_{3}$.

We claim that the resulting algebra $\widehat{E}_{3}$ is the quotient algebra $\left(\widehat{A}_{2} \oplus\right.$ $\left.\widehat{A}_{1}\right) /\left(K_{1}-K_{2}\right)$, where the ideal in the denominator is the difference between the two central terms $K_{1}$ and $K_{2}$. This retains only states which have levels satisfying $k_{1}=k_{2}$, ensuring there is only a single level $k$. Let $\alpha_{1}$ and $\alpha_{2}$ denote the roots of $A_{2}$ and $\beta_{1}$ the root of $A_{1}$. We then introduce new simple roots $\alpha_{0}$ and $\beta_{0}$, defined as

$$
\alpha_{0}=-\left(\alpha_{1}+\alpha_{2}\right)+\delta, \quad \beta_{0}=-\beta_{1}+\delta,
$$

where both new simple roots make use of the unique imaginary root $\delta$. The inner product $\alpha_{0} \cdot \beta_{0}$ vanishes and the Dynkin diagram of $\widehat{E}_{3}$, given in figure 3 , coincides with that of $\widehat{A}_{2} \oplus \widehat{A}_{1}$. Note, however, that the simple roots of $\widehat{E}_{3}$ are not all linearly independent, but satisfy the constraint

$$
\alpha_{0}+\alpha_{1}+\alpha_{2}=\beta_{0}+\beta_{1} .
$$

Representations of $\widehat{E_{3}}$ are products of one representation each from $A_{2}$ and $A_{1}$, constrained to have the same level.

Similarly, $\widehat{E}_{2}=\left(\widehat{A_{1}} \oplus \widehat{u(1)}\right) /\left(K_{1}-K_{u(1)}\right)$. Because of the $u(1)$ factor there is no Dynkin diagram, and the closest statement analogous to the constraint (3.28) is just that the two imaginary roots are identified by the quotient. 


\section{The loop algebra $\widehat{E}_{9}$}

The $\widehat{\mathbf{E}}_{\mathbf{N}}$ sequence of configurations is well-defined for all $N \geq 0$. We have already considered the cases $N \leq 8$ in some detail. In this subsection we will examine the configuration $\widehat{\mathbf{E}}_{\mathbf{9}}$ and will describe concretely the algebra $\widehat{E}_{9}$ it realizes. The brane configuration $\mathbf{A}^{8} \mathbf{B C B C}$ we are now identifying with $\widehat{\mathbf{E}}_{\mathbf{9}}$ was first considered in [9], where it was obtained by bringing together two groups of six branes, each defining a $\mathrm{D}_{4}$ configuration.

As can be seen from (3.8), the monodromy is $K\left(\widehat{\mathbf{E}}_{\mathbf{9}}\right)=\mathbb{1 1}$. Due to the trivial monodromy, a string of any charge can wind around the configuration and come back to itself. Hence $\widehat{\mathbf{E}}_{\mathbf{9}}$ supports two independent imaginary root junctions $\delta_{1}$ and $\delta_{2}$, having zero intersection with all roots including themselves and each other, which can be taken to have any linearly independent charges.

We have discussed how $\mathbf{E}_{\mathbf{8}}$ can be enhanced to either of two equivalent configurations $\mathbf{E}_{\mathbf{9}}=\mathbf{A}^{8} \mathbf{B C C}$ and $\widehat{\mathbf{E}}_{8}=\mathbf{A}^{7} \mathbf{B C C X} \mathbf{X}_{[3,1]}$. As depicted in figure 4 , we can visualize $\widehat{\mathbf{E}}_{\mathbf{9}}$ as the $\mathbf{E}_{\mathbf{8}}$ configuration with both of these enhancements. The $\boldsymbol{\delta}_{i}$ are then the two imaginary roots associated with the two distinct affinizations of $\mathbf{E}_{\mathbf{8}}$. The first imaginary root $\boldsymbol{\delta}_{1}$ can be taken to be a $(-1,0)$ loop around $\mathbf{E}_{\mathbf{8}}$ and the $[3,1]$ brane, while the second root $\boldsymbol{\delta}_{2}$ can be taken to be a $(3,1)$ loop around $\mathbf{E}_{\mathbf{8}}$ and the $[1,0]$ brane, both counterclockwise.

It should be noted that $\boldsymbol{\delta}_{1}$ can be made to pass through the $\mathbf{A}$ brane and $\boldsymbol{\delta}_{2}$ can be made to pass through $\mathbf{X}_{[\mathbf{3 , 1}]}$, as string segments can pass through a brane of the same charges without effect. We can thus deform to a presentation where both imaginary roots surround the full configuration, making it manifest that they do not intersect. For any given junction $\mathbf{J}$ there will be two levels $k_{i}=-\left(\mathbf{J}, \boldsymbol{\delta}_{i}\right)$ and two junction grades $n_{i}$ given by the number of prongs on the enhancing $\mathbf{A}$ and $\mathbf{X}_{[\mathbf{3}, \mathbf{1}]}$ branes.

The natural candidate for $\widehat{E}_{9}$ is the double loop algebra of $E_{8}$ with two central extensions. Since $E_{9}$ is the loop algebra of $E_{8}$, the double loop algebra in question is the loop algebra of $E_{9}$. While $E_{9}$ already has a grade and a central element, forming the loop algebra of $E_{9}$ will 


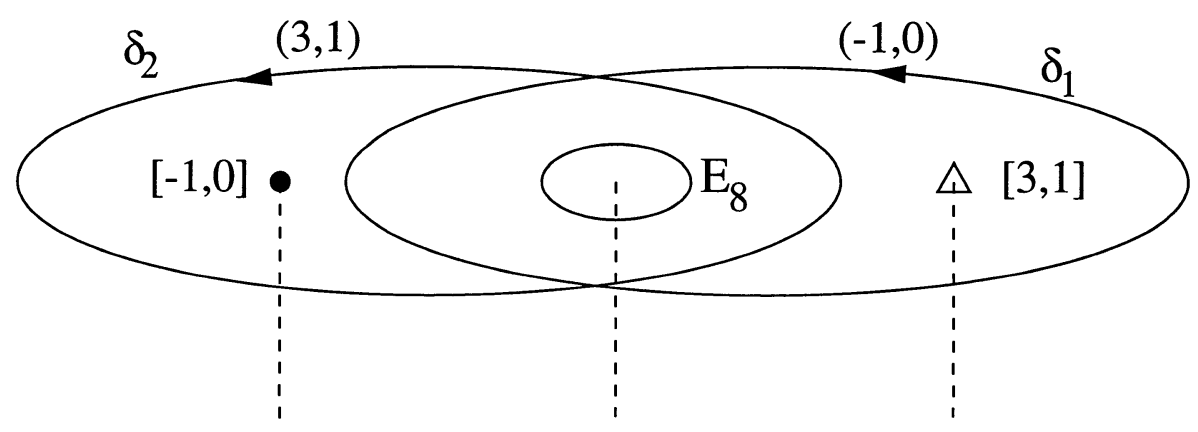

Figure 4: $\widehat{\mathbf{E}}_{\mathbf{9}}$ viewed as an enhancement of $\mathbf{E}_{\mathbf{8}}$ (grey oval) by branes with charges $[-1,0]$ and $[3,1]$. Both imaginary roots are presented as loops with certain charges.

introduce a new grade for every generator except the central one, and then a second nontrivial central extension can be added. Hence the algebra consists of the generators $\left\{T_{n_{1}, n_{2}}^{a}, K_{1}, K_{2}, D_{1}, D_{2}\right\}$ obeying the commutation relations

$$
\begin{aligned}
{\left[T_{n_{1}, n_{2}}^{a}, T_{m_{1}, m_{2}}^{b}\right] } & =f_{c}^{a b} T_{n_{1}+m_{1}, n_{2}+m_{2}}^{c}+\kappa^{a b}\left(n_{1} \delta_{n_{1}+m_{1}} K_{1}+n_{2} \delta_{n_{2}+m_{2}} K_{2}\right) \\
{\left[D_{i}, T_{n_{1}, n_{2}}^{a}\right] } & =-n_{i} T_{n_{1}, n_{2}}^{a} \\
{\left[K_{i}, T_{n_{1}, n_{2}}^{a}\right] } & =\left[K_{i}, D_{j}\right]=\left[D_{1}, D_{2}\right]=0
\end{aligned}
$$

where $a, b, c$ label the adjoint of $E_{8},\left\{f_{c}^{a b}\right\}$ are the $E_{8}$ structure constants, and $\kappa^{a b}$ is the $E_{8}$ Killing form. Just as a loop algebra is the algebra of maps $S^{1} \rightarrow \mathcal{G}$ for $\mathcal{G}$ finite and admits a central extension, the above algebra is algebra of maps $T^{2} \rightarrow E_{8}$ with two central extensions; we have

$$
T_{n_{1}, n_{2}}^{a}=T^{a} \otimes z_{1}^{n_{1}} \otimes z_{2}^{n_{2}}, \quad D_{i}=-z_{i} \frac{\partial}{\partial z_{i}},
$$

with $\left\{T^{a}\right\}$ the $E_{8}$ generators and the coordinates $z_{1}, z_{2}$ parameterizing the two circles.

Note that $\widehat{E}_{9}$ is not a Kac-Moody algebra, as it does not have a Cartan matrix. The necessary conditions on Cartan matrices of KacMoody algebras correspond to restrictions on the simple roots, including $\left(\alpha_{i}, \alpha_{i}\right)=2$ and $\left(\alpha_{i}, \alpha_{j}\right) \leq 0, i \neq j$. The $\delta_{i}$ are unacceptable as simple roots since $\left(\delta_{i}, \delta_{i}\right)=0$. An attempt to find two new simple roots 
in the spirit of affine algebras leads to $\alpha_{0}^{(i)}=\delta_{i}+\theta_{<}$, with $\theta_{<}$the lowest root of the $E_{8}$ adjoint. However we find that $\alpha_{0}^{(1)} \cdot \alpha_{0}^{(2)}=+2$, violating the second condition. There is no basis of simple roots simultaneously satisfying both. It is a nontrivial fact that the usual loop algebras with central extension of finite cases are equivalent to Kac-Moody algebras with an affine Cartan matrix. For the double loop algebra, there is no such correspondence.

We now proceed to examine the intersection form for $\widehat{\mathbf{E}}_{9}$. Consider an arbitrary junction $\mathbf{J}$ with asymptotic charges $(p, q)$, as depicted in figure 5 .

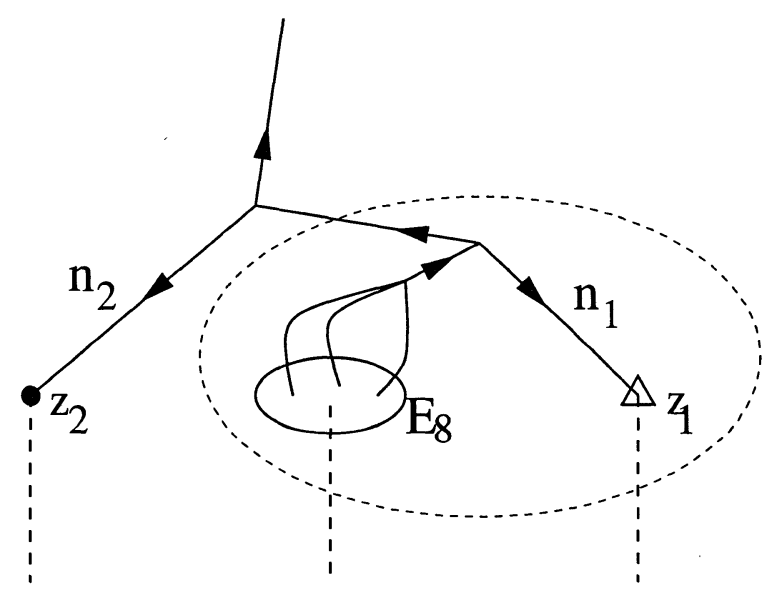

Figure 5: Calculating the intersection form for $\widehat{E}_{9}$.

First consider the sub-junction on the $\widehat{\mathbf{E}}_{\mathbf{8}}$ sub-configuration, as enclosed by the dashed line. The self-intersection of this piece, denoted $\left(\mathbf{J}_{9}\right)^{2}$, is given by (3.24) with $n_{1}$ in place of $n$, and charges $\left(p+n_{2}, q\right)$. The total self-intersection $\mathbf{J}^{2}$ is then the sum of $\left(\mathbf{J}_{9}\right)^{2},-\left(n_{2}\right)^{2}$ for the prongs on the $[1,0]$ brane, and a determinant giving the contribution at the intersection point:

$$
\begin{aligned}
\mathbf{J}^{2} & =\left(\mathbf{J}_{9}\right)^{2}-\left(n_{2}\right)^{2}+\operatorname{det}\left(\begin{array}{cc}
p & n_{2} \\
q & 0
\end{array}\right), \\
& =-\left(\lambda_{8} \cdot \lambda_{8}+2 n_{1} k_{1}\right)+f_{E_{8}}\left(p+n_{2}, q\right)-n_{2}^{2}-q n_{2} .
\end{aligned}
$$

The levels of $\mathbf{J}$ are given in terms of asymptotic charges by

$$
k_{1}=-q, \quad k_{2}=3 q-p .
$$


Solving for $p$ and $q$ in terms of the levels $k_{1}$ and $k_{2}$, and substituting the explicit form of the $E_{8}$ quadratic form we obtain

$$
\mathbf{J}^{2}=-\left(\lambda_{8} \cdot \lambda_{8}+2 n_{1} k_{1}+2 n_{2} k_{2}\right)+\left(k_{1}^{2}+k_{1} k_{2}+k_{2}^{2}\right) .
$$

The first group of terms is identified (up to shifts in grades) with the $\widehat{E}_{9}$ length squared of the associated weight vector. The two levels and grades enter symmetrically in the result. Note that while for the loop algebras of finite algebras one linear combination of asymptotic charges is determined by the level, here both asymptotic charges are determined by specifying the two levels. Thus $\widehat{\mathbf{E}}_{\mathbf{9}}$ is the only configuration we have examined where the algebraic properties of the associated weight vector completely specify a junction. It seems clear that to have double loop algebras we need configurations of branes with unit monodromy.

There are twelve branes in $\widehat{\mathbf{E}}_{\mathbf{9}}$, and it is possible to show that the monodromy of fewer then twelve branes is necessarily nontrivial ${ }^{3}$. Consider the commutator subgroup $G_{C} \subset G=S L(2, \mathbf{Z})$ which is the normal subgroup generated by commutators $g h g^{-1} h^{-1}, g, h \in G$. The quotient $G / G_{C}$ is abelian and is in fact the cyclic group $\mathbf{Z}_{12}$. $S L(2, \mathbf{Z})$ is generated by two elements $S$ and $U(=S T)$ which satisfy $S^{2}=U^{3}=-\mathbb{1}$, and then

$$
\left\{\mathbb{1},-U^{2} S, U, S, U^{2}, U S,-\mathbb{1}, U^{2} S,-U,-S,-U^{2},-U S\right\} .
$$

is a set of representatives from each coset of $G / G_{C}$. It is readily verified that this is isomorphic to the (additive) cyclic group of twelve elements, ordered $\{0,1,2, \ldots 11\}$. Consider now the canonical homomorphism $\mu$ : $S L(2, \mathbf{Z}) \rightarrow \mathbf{Z}_{12}$ defined by $\mu(g)=g G_{C}$. Note that $\mu\left(h g h^{-1}\right)=\mu(g)$, and since the monodromy of any 7 -brane is conjugate to that of a single A-brane, $K_{A}=\left(\begin{array}{cc}1 & -1 \\ 0 & 1\end{array}\right)=-U^{2} S, \mu$ maps it to $1 \in \mathbf{Z}_{12}$, and maps the overall monodromy of $N 7$-branes to $N(\bmod 12) \in \mathbf{Z}_{12}$. As $\mu(\mathbb{1})=0$, only products of $N=12 k 7$-brane monodromies for some $k \in \mathbf{Z}$ can multiply to the identity in $S L(2, \mathbf{Z})$. Only multiples of twelve branes can possess unit monodromy.

The twelve branes making up the elliptically fibered form of the ninth del Pezzo surface $\mathcal{B}_{9}$, also known as $\frac{1}{2} K 3$, are precisely those of $\widehat{\mathbf{E}}_{9}$. Two such $\widehat{\mathbf{E}}_{\mathbf{9}}$ configurations define a complete elliptically fibered

\footnotetext{
${ }^{3}$ We are grateful to Don Zagier for demonstrating this to us.
} 
K3. The global properties of the bases of these elliptic fibrations place additional constraints on the lattice of junctions and thus the algebra, as we shall explore in section 7. In particular, loops around the total configuration of branes on the base of either $\mathcal{B}_{9}$ or $\mathrm{K} 3$ are actually trivial since the base is a sphere.

Note that $\widehat{\mathbf{E}}_{\mathbf{N}}$ configurations with $N>9$ have the monodromy of $(N-9)$ [1,0]-branes. This is because the monodromy of the $\widehat{\mathbf{E}}_{\mathbf{9}}$ subconfiguration is one, and only the contribution of the additional $(N-9)$ $[1,0]$-branes is visible. This does not mean the algebra is elementary. The next term in the series, for example is $\widehat{\mathbf{E}}_{10}$ and as its name suggests we associate it with $\widehat{E}_{10}$, the loop algebra of $E_{10}$. While all $E_{N}$ algebras are Kac-Moody, for $N \geq 9$ their loop extensions $\widehat{E}_{N}$ are not.

\section{Indefinite enhancement: $f(\mathbf{z})>1$}

We have seen in the previous section that it is possible to realize infinite dimensional algebras such as $\widehat{E}_{9}$ that are not Kac-Moody. On the other hand, it is also straightforward to find cases of infinite-dimensional KacMoody algebras that are not affine. These are called indefinite KacMoody algebras on account of the indefinite signature of their Cartan matrix. We shall see that indefinite Kac-Moody algebras appear on 7-branes when one adds to a finite algebra configuration an enhancing brane $\mathbf{Z}$ with charges satisfying $f(\mathbf{z})>1$. We will identify explicitly the brane configurations giving the hyperbolic extension of the exceptional series $E_{N}^{H}$, including $E_{10}=E_{8}^{H}$. The exceptional series $E_{N}$ for $N>10$ consists of Lorentzian Kac-Moody algebras that are not hyperbolic. We also describe a few other indefinite examples, including a class of algebras with an overcomplete basis of simple roots with a constraint, and the complete series of strictly hyperbolic algebras of rank 2 .

For enhancement to an affine algebra from a simple one, we required $f(\mathbf{z})=1$, and the $n$ dependence of equation (2.9) dropped out. As a result, any Weyl orbit satisfying $\lambda \cdot \lambda=-\mathbf{J}^{2}$ appeared at all grades. On the other hand when $f(\mathbf{z})>1, f(\mathbf{z})-1$ is positive, and thus as $|n|$ increases in (2.9), the length-squared of acceptable Weyl orbits for a given $\mathbf{J}^{2}$ grows. Unlike the affine root systems, which were infinite but repeated the same set of finite root vectors at all grades, the in- 
definite root systems grow uncontrollably, with larger finite weights at each grade. In addition, in contrast to the affine case there are clearly solutions with $\mathbf{J}^{2}>0$. In fact, typically for a given positive even $\mathbf{J}^{2}$ there exist infinitely many roots, with arbitrarily large $|n|$ and $\lambda \cdot \lambda$.

Since indefinite Cartan matrices are invertible, the intersection form for the associated brane configurations has the same elementary decomposition into Lie algebra and asymptotic charge blocks as the finite case, as equation (2.4). Once again $\lambda \cdot \lambda$ is defined by the inverse Cartan matrix, and $f(p, q)$ is the contribution to the intersection from asymptotic charges, as in (2.5). Notice how this is much simpler than the affine case.

\subsection{Hyperbolic exceptional algebras}

The Dynkin diagram of a hyperbolic algebra contains only affine and finite subdiagrams, and therefore hyperbolic algebras are the simplest kind of indefinite Kac-Moody algebras. Given a finite algebra $\mathcal{G}$ the canonical hyperbolic extension $\mathcal{G}^{H}$ is obtained by attaching two new nodes to the Dynkin diagram: the first node is the one giving the affine extension $\widehat{\mathcal{G}}$, and the second node is attached to the first and to no other node [18]. Perhaps the most familiar hyperbolic algebra is $E_{10}=E_{8}^{H}$, which has as root lattice the unique even unimodular lattice with Lorentzian $(9,1)$ signature. As discussed in the introduction, its possible relevance to string theory has been the subject of much interest. Hyperbolic algebras have been studied somewhat by mathematicians but, their root multiplicities and representation theory remain fairly mysterious.

Let us begin with an example. Consider $\mathbf{E}_{\mathbf{8}}=\mathbf{A}^{7} \mathbf{B C C}$, which can be enhanced to various algebras of rank 9 by adding a single brane. The charge quadratic form, as given in table (2), is

$$
f_{E_{8}}(p, q)=p^{2}-5 p q+7 q^{2} \text {. }
$$

Charges $\mathbf{z}=[p, q]$ giving $f(\mathbf{z})=1$ enhance to $\mathbf{E}_{\mathbf{9}} \cong \widehat{\mathbf{E}}_{\mathbf{8}}$. The next smallest possibility is $f(\mathbf{z})=3$, which arises for charges [1,1], among others. The monodromy matrix $K$ of this configuration satisfies $\operatorname{Tr} K=$ 4. Since $f(\mathbf{z})>1$, this algebra will be indefinite. We search for a new 
simple root, so we focus on junctions with $\mathbf{J}^{2}=-2$. Using (2.9), such junctions $\mathbf{J}=(n, \lambda)$ obey

$$
\lambda \cdot \lambda=2+2 n^{2} .
$$

At $n=1$ we must have $\lambda \cdot \lambda=4$, which is satisfied only by the Weyl orbit with highest weight $\omega^{1}$. This Weyl orbit contains 2160 weight vectors, and is the largest Weyl orbit of the $\mathbf{3 8 7 5}$. We pick the new simple root $\boldsymbol{\alpha}_{0}=\left(1,-\omega^{1}\right)$, which attaches an extra node to $E_{8}$ giving us the Dynkin diagram $T_{5,4,2}$, associated to the hyperbolic algebra $E_{7}^{H}$. In the table below we give the length-squared $\lambda \cdot \lambda$ for the Weyl orbits of the real roots and the first few sets of imaginary roots, for the lowest grades.

\begin{tabular}{|c|c|c|c|c|}
\hline$\lambda \cdot \lambda$ & $\mathbf{J}^{2}=-2$ & $\mathbf{J}^{2}=0$ & $\mathbf{J}^{2}=2$ & $\mathbf{J}^{2}=4$ \\
\hline$|n|=0$ & 2 & 0 & - & - \\
$|n|=1$ & 4 & 2 & 0 & - \\
$|n|=2$ & 10 & 8 & 6 & 4 \\
$|n|=3$ & 20 & 18 & 16 & 14 \\
\hline
\end{tabular}

We could have obtained $E_{7}^{H}$ by enhancing $\widehat{E}_{7}$ as well. Indeed, starting with $\mathbf{E}_{\boldsymbol{7}}=\mathbf{A}^{6} \mathbf{B C C}$ we enhance to $\widehat{\mathbf{E}}_{\boldsymbol{7}}=\mathbf{A}^{6} \mathbf{B C C X} \mathbf{X}_{[\mathbf{3}, \mathbf{1}]}$ and get the hyperbolic configuration by adding yet another $[3,1]$ brane: $\mathbf{E}_{\mathbf{7}}^{\mathbf{H}}=\mathbf{A}^{6} \mathbf{B C C}\left(\mathbf{X}_{[\mathbf{3}, \mathbf{1}]}\right)^{2}$. The first $[3,1]$ brane creates a node for the root junction $\boldsymbol{\alpha}_{0}$, a junction that has a single prong on that brane. Once we add the second $[3,1]$ brane we obtain a new junction joining the two $[3,1]$ branes, representing a new simple root $\boldsymbol{\alpha}_{-1}$ producing a new node that only attaches to the first added node. Therefore in general we have the configurations

$$
\mathbf{E}_{\mathbf{N}}^{\mathbf{H}}=\mathbf{A}^{N-1} \mathbf{B C C}\left(\mathbf{X}_{[3,1]}\right)^{2},
$$

realizing the hyperbolic Lie algebra $E_{N}^{H}$ for $N \leq 8$, and "hyperbolic" enhancements of the non-Kac-Moody loop algebras discussed in section 4 for $N>8$. The monodromy is

$$
K\left(\mathbf{E}_{\mathbf{H}}^{\mathbf{N}}\right)=\left(\begin{array}{cc}
4 & 27-4 N \\
1 & 7-N
\end{array}\right)
$$

All these configurations for $N \leq 7$ can also be obtained by adding a single brane to a configuration with a finite Lie algebra of rank $N+1$, as we saw above for the case of $\mathbf{E}_{\mathbf{8}} \rightarrow \mathbf{E}_{\mathbf{7}}^{\mathbf{H}}$. 


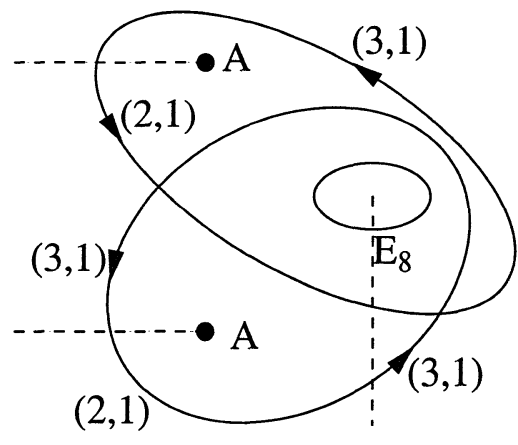

(a)

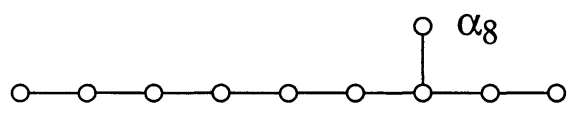

$\alpha_{-1} \alpha_{0}$

Figure 6: (a) The $\mathbf{E}_{\mathbf{1 0}}$ configuration viewed as an enhancement of $\mathbf{E}_{8}$ (grey oval) by two $[1,0]$ branes, with the two intersecting imaginary roots indicated. The only nonzero contribution to the intersection comes from where the $(2,1)$ string segment crosses the $(3,1)$ one. (b) The $E_{10}$ Dynkin diagram.

The configuration $\mathbf{E}_{\mathbf{8}}^{\mathbf{H}}$ as defined in (5.3) realizes the algebra $E_{8}^{H}=$ $E_{10}$, and is equivalent to a member of the $\mathbf{E}_{\mathbf{N}}$ series, $\mathbf{E}_{\mathbf{1 0}}=\mathbf{A}^{9} \mathbf{B C C}$. We use this presentation, illustrated in figure 6 , to highlight the difference between the algebras $E_{10}$ and $\widehat{E}_{9}$. The latter, as explained in section 4 , can be thought of as $E_{8}$ with two additional imaginary roots which have zero intersection with all roots, including each other. On the other hand, $E_{10}$ can be thought of as $E_{8}$ with two new roots $u_{i}$ that are "imaginary" in the sense that they have $u_{i} \cdot u_{i}=0$ and $\left(u_{i}, \alpha\right)=0$ for all roots $\alpha$ of $E_{8}$, but which also have nonzero intersection with each other: $u_{1} \cdot u_{2}=1$. Then the affine root is $\alpha_{0}=-u_{1}+\theta$, and the hyperbolic root is $\alpha_{-1}=u_{1}+u_{2}$ [18].

The brane picture makes this manifest. Either one of the two enhancing $\mathbf{A}$ branes alone would produce $\mathbf{E}_{\mathbf{9}}$ with an imaginary root; these two $\boldsymbol{\delta}_{i}$ correspond to the two $u_{i}$. When both enhancing branes are present, we still have both imaginary roots. Note that the loops intersect nontrivially; at one of their crossing points their charges coincide but at the other one they do not. Having nontrivial intersection, it is impossible to have any of these loops encircle the whole configuration. Indeed the total monodromy does not allow such loop junction, consistent with $E_{10}$ not being a loop algebra. 


\subsection{Algebras with two enhanced simple roots}

Another notable set of enhancements of exceptional finite algebras is characterized by more than one Weyl orbit having the value of $\lambda \cdot \lambda$ necessary to solve (2.9) at $n=1$. It is then not possible to choose a single new simple root that spans the entire set of new roots, since if it is chosen from one of the Weyl orbits some roots from the other will be missed. These indefinite algebras have an interpretation: they are the enhancements of affine semisimple algebras.

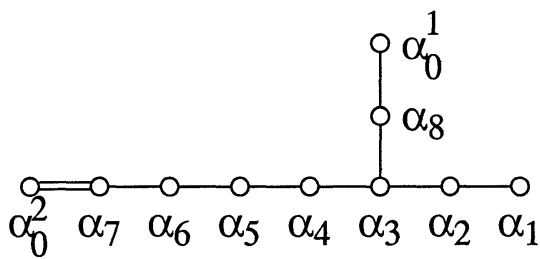

(a)

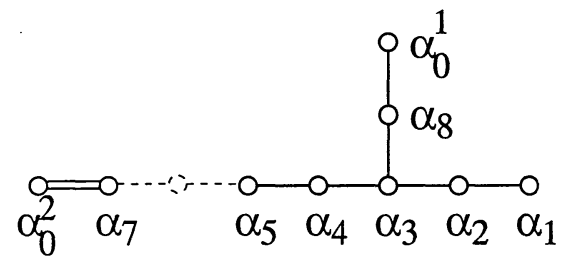

(b)

Figure 7: (a) The Dynkin diagram resulting from adding both new simple roots to $E_{8}$. (b) With the node corresponding to $\alpha_{6}$ removed, Figure 7 becomes the Dynkin diagram of the affine version of $E_{6} \oplus A_{1}$.

We give an example, again with $\mathbf{E}_{\mathbf{8}}$. Adding a $[0,1]$ brane gives $f(0,1)=7$, the next smallest value after $f=3$. Then at $n=1$ we can have new real roots with $\lambda \cdot \lambda=8$. There are two $E_{8}$ Weyl orbits with this length-squared, with highest weight vectors $\omega^{8}$ and $2 \omega^{7}$. We cannot choose a single simple root, but instead have the candidates

$$
\boldsymbol{\alpha}_{0}^{1}=\left(1,-\omega^{8}\right), \quad \boldsymbol{\alpha}_{0}^{2}=\left(1,-2 \omega^{7}\right) .
$$

We have already encountered algebras where a single new simple root was not sufficient, but instead two new simple roots and a constraint were necessary, in the case of $\widehat{E}_{3}$, and this is no coincidence. Let us calculate the mutual intersection $\left(\boldsymbol{\alpha}_{0}^{1}, \boldsymbol{\alpha}_{0}^{2}\right)$. In terms of explicit basis junctions as in (2.8), we have

$$
\boldsymbol{\alpha}_{0}^{1}=-\mathbf{z}+\boldsymbol{\omega}^{q}-\boldsymbol{\omega}^{8}, \quad \boldsymbol{\alpha}_{0}^{2}=-\mathbf{z}+\boldsymbol{\omega}^{q}-2 \boldsymbol{\omega}^{7},
$$

and then we calculate

$$
\begin{aligned}
\left(\boldsymbol{\alpha}_{0}^{1}, \boldsymbol{\alpha}_{0}^{2}\right) & =-1+2\left(\boldsymbol{\omega}^{7}, \boldsymbol{\omega}^{8}\right)+\left(\boldsymbol{\omega}^{q}, \boldsymbol{\omega}^{q}\right) \\
& =-1-6+f(0,1) \\
& =0
\end{aligned}
$$


and so we can take this enhanced algebra to be the Kac-Moody algebra determined by the Dynkin diagram in Fig. 7(a), with an additional constraint on the set of simple roots. One can compute that in $E_{8}$

$$
\omega^{8}-2 \omega^{7}=\alpha_{1}+2 \alpha_{2}+3 \alpha_{3}+2 \alpha_{4}+\alpha_{5}+2 \alpha_{8}-\alpha_{7} .
$$

It follows from this relation and (5.5) that

$$
\alpha_{0}^{1}+\alpha_{1}+2 \alpha_{2}+3 \alpha_{3}+2 \alpha_{4}+\alpha_{5}+2 \alpha_{8}=\alpha_{0}^{2}+\alpha_{7} .
$$

It is possible to express this algebra as a further enhancement of an affine semisimple algebra. To see this, first remove the node corresponding to $\alpha_{6}$ in the Dynkin diagram, giving us Fig. 7(b). Without the two enhancing nodes associated to $\alpha_{0}^{1}$ and $\alpha_{0}^{2}$, this is just the Dynkin diagram of the semisimple algebra $E_{6} \oplus A_{1}$. The two enhancing nodes then produce the affine enhancement, $\widehat{E_{6} \oplus A_{1}}=\widehat{E_{6}} \oplus \widehat{A_{1}} / \sim$, just like the affinization $E_{3} \rightarrow \widehat{E}_{3}$. From this point of view each enhancing root satisfies $\alpha_{0}^{i}=-\theta_{i}+\delta$, where the $\theta_{i}$ are the highest roots of $E_{6}$ and $A_{1}$ respectively and $\delta$ is the same in both cases. Thus one can relate the two enhancing roots by

$$
\delta=\alpha_{0}^{1}+\theta_{E_{6}}=\alpha_{0}^{2}+\theta_{A_{1}},
$$

and using the $E_{6}$ and $A_{1}$ marks we find expressions for the $\theta_{i}$ in terms of the $\alpha_{i}$ appearing in the Dynkin diagram. This gives exactly (5.9).

Thus Lie algebraically, the algebra we obtain by adding the enhancing nodes to $E_{8}$ is the same as what we obtain starting with $E_{6} \oplus A_{1}$, affinizing, and then further enhancing by restoring the node $\alpha_{6}$. We can think of this as affinizing $E_{8}$ with respect to its $E_{6} \oplus A_{1}$ subalgebra. In that sense, the new direction in root space corresponds to the addition of a root $\delta$, which satisfies $(\delta, \delta)=0$ and $\left(\delta, \alpha_{i}\right)=0$ for all roots in the $E_{6} \oplus A_{1}$ subalgebra, but must satisfy $\left(\delta, \alpha_{6}\right)=-1$ so that $\left(\alpha_{0}^{i}, \alpha_{6}\right)=0$.

This pattern of enhancements repeats itself for the other members of the $\mathbf{E}_{\mathbf{N}}$ series of configurations. In general, the finite $E_{N}$ algebra can be made to enhance to an indefinite algebra which is an enhancement of $\widehat{E}_{N-2} \oplus \widehat{A}_{1} / \sim$. Alternately, this can be thought of as starting with $E_{N}$ and affinizing with respect to the semisimple subalgebra $E_{N-2} \oplus$ $A_{1}$. The appropriate values of $f(\mathbf{z})$ are the next-smallest after those enhancing to $\mathbf{E}_{\mathbf{N}-\mathbf{1}}^{\mathrm{H}}$. In each case we find two Weyl orbits satisfying 
(2.9) at $n=1$, giving two linearly dependent enhancing simple roots. We write the Cartan matrix by including both new simple roots, and imposing a constraint on the set of roots. Let $c^{i}$ and $d^{i}$ be the extensions of the marks of $E_{N-2}$ and $A_{1}$ to $E_{N}$, defined as follows. Let the $\alpha_{i}$ be the simple roots of $E_{N}$. Then if $\alpha_{i}$ is a simple root of the $E_{N-2}$ subalgebra, $c^{i}$ is the mark of that $E_{N-2}$ root, otherwise $c^{i}=0$, and likewise for $d^{i}$ and the $A_{1}$ subalgebra. Then the constraint on the simple roots for the enhanced algebra is

$$
\alpha_{0}^{E_{N-2}}+\sum_{i=1}^{N} c^{i} \alpha_{i}=\alpha_{0}^{A_{1}}+\sum_{i=1}^{N} d^{i} \alpha_{i},
$$

where $\alpha_{0}^{E_{N-2}}$ and $\alpha_{0}^{A_{1}}$ are the two new simple roots associated to affinizing the two different simple subalgebras.

\subsection{Rank 2 hyperbolic algebras}

There is a final interesting family of enhancements we mention before ending this section. $\mathbf{E}_{\mathbf{1}}$ can be enhanced to configurations realizing any simply-laced rank 2 algebra with Cartan matrix

$$
A=\left(\begin{array}{cc}
2 & -a \\
-a & 2
\end{array}\right)
$$

for any positive integer $a$. For $\mathbf{E}_{\mathbf{1}}$, the quadratic form is $f(p, q)=$ $\frac{1}{8}\left(p^{2}+2 p q-7 q^{2}\right)$. Junctions only arise for $p+q=0(\bmod 2)$ due to the charges of BCC. Consider adding a brane with charges $\mathbf{z}=[ \pm 2 a-1,1]$. We obtain $f(\mathbf{z})=\frac{1}{2} a^{2}-1$, and hence for $\mathbf{J}^{2}=-2$ at $n=1$ we find $\lambda \cdot \lambda=\frac{1}{2} a^{2}$. This is the length-squared of the Weyl orbit of $s u(2)$ with dominant weight given by Dynkin label $a$. We are thus led to choose $\boldsymbol{\alpha}_{0}=(1,-a \omega)$, and $\left(\boldsymbol{\alpha}_{0}, \boldsymbol{\alpha}\right)=-a$.

For $a=0$ this enhances to $A_{1} \oplus A_{1}$, and for $a=1$ we find $A_{2}$. The value $a=2$ gives the affine algebra $\widehat{A_{1}}$, and $a \geq 3$ gives the complete series of strictly hyperbolic simply laced algebras of rank 2 . Imaginary roots appear for $a \geq 2$. All of these configurations only support asymptotic charges with $p+q=0(\bmod 2)$. All charges only become possible when an enhancing brane is added with $[p, q]$ labels that do not satisfy this condition. 


\begin{tabular}{|c|c|c|c|c|c|}
\hline $\mathcal{G}$ & Config & Branes & $f(\mathbf{z})$ & $\mathcal{G}^{e n h}$ & Enh. branes \\
\hline$A_{N}$ & $\mathbf{H}_{\mathbf{N}}$ & $\mathbf{A}^{N+1} \mathbf{C}$ & $\begin{array}{c}-1 / N \\
(N-3) /(N+1) \\
(2 N-7) /(N+1)\end{array}$ & $\begin{array}{l}A_{N+1} \\
D_{N+1} \\
E_{N+1}\end{array}$ & $\begin{array}{c}\mathbf{A H}_{\mathbf{N}} \\
\mathbf{H}_{\mathbf{N}} \mathbf{X}_{[3,1]} \\
\mathbf{H}_{\mathbf{N}} \mathbf{X}_{[4,1]}\end{array}$ \\
\hline$D_{N}$ & $\mathrm{D}_{\mathrm{N}}$ & $\mathbf{A}^{N} \mathbf{B C}$ & $\begin{array}{c}0 \\
(N-4) / 4 \\
\end{array}$ & $\begin{array}{l}D_{N+1} \\
E_{N+1} \\
\end{array}$ & $\begin{array}{l}\mathrm{AD}_{\mathrm{N}} \\
\mathrm{D}_{\mathrm{N}} \mathrm{C} \\
\end{array}$ \\
\hline$A_{1}$ & $\mathbf{E}_{1}$ & $\mathrm{BCC}$ & $a^{2} / 2-1$ & $\tilde{A}_{2}^{(a)}$ & $E_{1} X_{[2 a-1,1]}$ \\
\hline$D_{4}$ & $\mathrm{D}_{4}$ & $\mathrm{~A}^{4} \mathrm{BC}$ & 0 & $D_{5}$ & $\mathbf{D}_{4} \mathbf{X}_{[\mathbf{p}, \mathbf{q}]}$ \\
\hline$D_{5}$ & $\begin{array}{c}\mathrm{D}_{5} \\
\cong \mathrm{E}_{5}\end{array}$ & $\mathrm{~A}^{5} \mathrm{BC}$ & $\begin{array}{c}0 \\
1 / 4 \\
1 \\
9 / 4 \\
\end{array}$ & $\begin{array}{l}D_{6} \\
E_{6} \\
\widehat{D}_{5} \\
E_{4}^{H} \\
\end{array}$ & $\begin{array}{c}\mathrm{AD}_{5} \\
\mathrm{D}_{5} \mathrm{C} \\
\mathrm{D}_{5} \mathrm{X}_{[1,2]} \\
\mathrm{D}_{5} \mathrm{X}_{[1,3]}\end{array}$ \\
\hline$E_{6}$ & $\mathbf{E}_{6}$ & $\mathrm{~A}^{5} \mathrm{BCC}$ & $\begin{array}{c}1 / 3 \\
1 \\
7 / 3\end{array}$ & $\begin{array}{l}E_{7} \\
\widehat{E}_{6} \\
E_{5}^{H}\end{array}$ & $\begin{array}{c}\mathbf{A E}_{6} \\
\mathrm{E}_{6} \mathbf{X}_{[3,1]} \\
\mathrm{E}_{6} \mathbf{X}_{[\mathbf{4 , 1}]}\end{array}$ \\
\hline$E_{7}$ & $\mathbf{E}_{7}$ & $\mathrm{~A}^{6} \mathrm{BCC}$ & $\begin{array}{c}1 / 2 \\
1 \\
5 / 2\end{array}$ & $\begin{array}{l}E_{8} \\
\widehat{E}_{7} \\
E_{6}^{H}\end{array}$ & $\begin{array}{c}\mathbf{A E}_{\mathbf{7}} \\
\mathrm{E}_{\mathbf{7}} \mathbf{X}_{[3,1]} \\
\mathrm{E}_{\mathbf{7}} \mathbf{X}_{[\mathbf{4}, \mathbf{1}]}\end{array}$ \\
\hline$E_{8}$ & $\mathbf{E}_{8}$ & $\mathrm{~A}^{7} \mathrm{BCC}$ & $\begin{array}{l}1 \\
3\end{array}$ & $\begin{array}{c}\widehat{E}_{8}=E_{9} \\
E_{7}^{H}\end{array}$ & $\begin{array}{c}\mathbf{A E}_{8} \\
\mathbf{E}_{8} \mathbf{X}_{[4,1]}\end{array}$ \\
\hline
\end{tabular}

Table 4: Finite algebras $\mathcal{G}$ and enhanced algebras $\mathcal{G}^{\text {enh }}$ obtained by adding a single $\mathbf{z}=[p, q]$ brane. The Dynkin diagram of $\tilde{A}_{2}^{(a)}$ has two nodes with $a$ lines joining them.

These cases only scratch the surface of the possible indefinite algebras arising on 7-branes. Ignoring for the moment that F-Theory compactifications permit only 24 7-branes, and considering only the algebraic properties of the monodromies, algebras of arbitrarily large rank will appear in our construction. Most of these algebras have no known relation to physics. We do not, however, expect all simply-laced algebras to arise. For example, recall that there was no way to enhance $D_{4}$ to $\widehat{D}_{4}$. This affine algebra has the simplest Dynkin diagram which contains four nodes each with a one line connecting to a single, fifth node. If this configuration appeared as a subdiagram in any larger algebra we would expect to be able to decouple branes and eventually reach $\widehat{D}_{4}$, so this must not occur. Thus any node in a Dynkin diagram 
arising on 7-branes must branch to at most three other nodes. In general the affine $\widehat{A}_{N}$ and $\widehat{D}_{N}$ series do not appear (except for $\widehat{A}_{4}=\widehat{E}_{4}$ and $\widehat{D}_{5}=\widehat{E}_{5}$ ), so presumably they do not arise as subdiagrams either. The complete classification of indefinite algebras realized on 7-branes is still an open problem. The various enhancements obtained in the last three sections are summarized in Table 4.

\section{$6 \quad S L(2, \mathbf{Z})$ Conjugacy Classes and Classification}

In the previous sections we explored the appearance of various infinitedimensional algebras on configurations of 7-branes. Which algebra appears is largely determined by the total monodromy of the brane configuration. Since the physical situation is invariant under global $S L(2, \mathbf{Z})$ transformations, the Lie algebra is not associated with a monodromy but rather with the conjugacy class of the monodromy. The monodromy is conjugated by this transformation, and as a result we expect that the equivalence classes of 7-brane configurations, and therefore the corresponding Lie algebras, are related to the conjugacy classes of $S L(2, \mathbf{Z})$. It is this relation that we explore in this section.

As we have commented, the equivalence class of the monodromy has the dominant role in determining the algebra, but we are aware of two other factors which must also be specified: the number of branes and the integer $\ell$ discussed in sec. 2. The number of branes must be specified because there can exist sub-configurations of branes with unit monodromy. As we have proven, such configurations must have some multiple of twelve branes, and specifying the total number of branes removes the ambiguity they create. The integer $\ell$ is invariant under global $S L(2, \mathbf{Z})$ and branch cut relocation, and measures whether there are constraints on the possible asymptotic charges. Configurations with the same monodromy and number of branes must still be inequivalent when $\ell$ differs. For almost all configurations, $\ell=1$, and all asymptotic charges can be realized.

Conjugacy classes of $S L(2, \mathbf{Z})$ are conveniently organized by their trace $t$. Classes with $|t|<2$ are known as elliptic, classes with $|t|=2$ parabolic and classes with $|t|>2$ hyperbolic. 
In [6], the finite Lie algebras arising on 7-branes were matched to the corresponding $S L(2, \mathbf{Z})$ conjugacy classes. It is instructive to see how the rank of these algebras is fixed by the monodromy. Consider for example, $t=0$, the representatives of the two conjugacy classes at this trace are $S$ and $-S$. As follows from the abelianization of $S L(2, \mathbf{Z})$ (see (4.6) and the discussion below it), the configurations realizing the overall monodromy which is conjugate to $S$ or $-S$ must contain $3(\bmod 12)$ and $9(\bmod 12) 7$-branes, respectively. In the simplest case of no more than 12 branes this yields algebras of rank $1\left(A_{1}\right)$ and $7\left(E_{7}\right)$, respectively. Similar arguments apply at other values of $t$.

There are two classes for each elliptic $t$, and the six inequivalent elliptic configurations were the three exceptional configurations $\mathbf{E}_{\mathbf{6}}, \mathbf{E}_{\mathbf{7}}$, $\mathbf{E}_{\mathbf{8}}$ and the Argyres-Douglas $\mathbf{H}_{\mathbf{0}}, \mathbf{H}_{\mathbf{1}}$ and $\mathbf{H}_{\mathbf{2}}$, all collapsible configurations leading to Kodaira singularities. There are an infinite number of classes at both $t=2$ and $t=-2$, and some of these correspond to configurations of mutually local branes $\mathbf{A}_{\mathbf{N}}(t=2)$ which are collapsible for all $N$, and $\mathbf{D}_{\mathbf{N}}$ configurations $(t=-2)$, which exist for all non-negative $N$ but are collapsible only for $N \geq 4$. These series fill an infinite number of parabolic conjugacy classes, but an infinite number still remain. Finally, the $\mathbf{E}_{\mathbf{N}}$ configurations could be extended to $0 \leq N \leq 5$, and the $\mathbf{H}_{\mathbf{N}}$ to $N \geq 3$. The cases $\mathbf{E}_{\mathbf{5}} \cong \mathbf{D}_{\mathbf{5}}$ and $\mathbf{H}_{\mathbf{3}} \cong \mathbf{D}_{\mathbf{3}}$ correspond to parabolic classes, while the rest are associated to hyperbolic classes of negative trace. At $t=-6$ we had the conjugacy class for $\mathbf{E}_{\mathbf{1}}$ and $\tilde{\mathbf{E}}_{\mathbf{1}}$, these configurations are inequivalent since $\ell\left(\mathbf{E}_{\mathbf{1}}\right)=2$ and $\ell\left(\tilde{\mathbf{E}}_{\mathbf{1}}\right)=1$. These results are summarized in table 5 .

Let us now explore how the infinite-dimensional algebras fit into this scheme. They can occupy the remaining parabolic and hyperbolic conjugacy classes. The number $H(t)$ of hyperbolic conjugacy classes at each value of $t$ cannot be determined by elementary methods as was done for the elliptic and parabolic cases in [6]. There one could relate the fixed point of the elements of an elliptic or parabolic class to a fixed point in the fundamental domain $\mathcal{F}$, but since the fixed points of hyperbolic classes are irrational real numbers, they cannot be mapped to $\mathcal{F}$. Instead, more sophisticated machinery is necessary to determine $H(t)$ for $|t|>2$.

One determines $H(t)$ by using the isomorphism between the conjugacy classes of $S L(2, \mathbf{Z})$ matrices of trace $t$ and equivalence classes of 
binary quadratic forms of discriminant $t^{2}-4$. This isomorphism associates the charge quadratic form with a given monodromy, as discussed in [6]. The number $H(t)$ of conjugacy classes in $S L(2, \mathbf{Z})$ with trace $t$ is

$$
H(t)=h_{+}\left(t^{2}-4\right),
$$

where $h_{+}(\widehat{d})$ denotes the number of equivalence classes of binary quadratic forms with discriminant $\widehat{d}$. Moreover, the equivalence classes of binary quadratic forms of discriminant $\widehat{d}$ are in one-to-one correspondence with the (strict) equivalence classes of ideals in a quadratic field of discriminant $\widehat{d}$. This relation allows us to determine $H(t)$ for arbitrary $t$. This process is somewhat technical, and is explained in the appendix. There table 7 lists the number of conjugacy classes and gives representatives for $-15 \leq t \leq 15$.

In section 3 we found configurations $\widehat{\mathbf{E}}_{\mathbf{N}}$ realizing affine versions of the exceptional algebras. We noted there that all $S L(2, \mathbf{Z})$ matrices $K$ with a unit eigenvalue have $t=\operatorname{Tr} K=2$, including the monodromy matrices $K\left(\widehat{\mathbf{E}}_{\mathbf{N}}\right)$ of the affine configurations:

$$
\operatorname{Tr} K\left(\widehat{\mathbf{E}}_{\mathbf{N}}\right)=2 \text {. }
$$

Thus all the affine algebras are associated to parabolic conjugacy classes. Representatives of the $t=2$ classes are $\left(\begin{array}{cc}1 & -n \\ 0 & 1\end{array}\right)$ with $n \in \mathbf{Z}$. From (3.8), we see that $\widehat{\mathbf{E}}_{\mathbf{N}}$ configurations correspond to classes with $n=N-9$.

At $N=1$, there are the two inequivalent configurations $\widehat{\mathbf{E}}_{\mathbf{1}}$ and $\widehat{\tilde{\mathbf{E}}}_{1}$ which are the affinizations of the corresponding inequivalent finite cases as explored in [6]. They share the same conjugacy class, and are distinguished by $\ell$. For $N=0$ we have the $\widehat{\tilde{\mathbf{E}}}_{0}$ configuration. Thus $\widehat{\mathbf{E}}_{\mathbf{N}}$ is defined for all $N \geq 0$. For $N>9$ the monodromy of this series coincides with that of $\mathbf{A}_{\mathbf{N - 1 0}}$, as noted at the end of section 4; the different series differ by a factor of $\widehat{\mathbf{E}}_{\mathbf{9}}$.

The series $\mathbf{E}_{\mathbf{N}}$ realizes infinite algebras for $N \geq 9$. For $N=9$ this is just the affine configuration $\mathbf{E}_{\mathbf{9}} \cong \widehat{\mathbf{E}}_{\mathbf{8}}$, while for $N \geq 10$ we have a series of infinite- dimensional exceptional algebras. From Table 2 we have

$$
\operatorname{Tr} K\left(\mathbf{E}_{\mathbf{N}}\right)=N-7,
$$


we see that for $N \geq 10$ the associated conjugacy classes are hyperbolic with positive trace.

In section 5, we found the hyperbolic exceptional configurations $\mathbf{E}_{\mathbf{N}}^{\mathbf{H}}$. Using (5.4) one can see

$$
\operatorname{Tr} K\left(\mathbf{E}_{\mathbf{N}}^{\mathbf{H}}\right)=11-N .
$$

At $t=3$, we find $H(3)=1$ and thus a single conjugacy class for $\mathbf{E}_{\mathbf{1 0}} \cong \mathbf{E}_{\mathbf{8}}^{\mathbf{H}}$. The other elements in the $\mathbf{E}_{\mathbf{N}}$ and $\mathbf{E}_{\mathbf{N}}^{\mathbf{H}}$ series are distinct, and correspondingly $H(t)>1$ for $3<t<10$ where both are defined. These configurations are displayed up to $t=7$ in Table 5 .

Section 5 discussed a few other configurations realizing infinitedimensional algebras which lie outside the scope of Table 5. The enhancement of $\mathbf{E}_{\mathbf{8}}$ to a configuration requiring two new simple roots, with Dynkin diagram given in figure 7 , appears at $t=8$. Since $\mathbf{E}_{\mathbf{1 5}}$ and $\mathbf{E}_{\mathbf{3}}^{\mathbf{H}}$ are already expected to be present as well, we anticipate that there are more than two conjugacy classes at $t=8$, and indeed $H(8)=4$. The corresponding enhancements of $\mathbf{E}_{\mathbf{7}}, \mathbf{E}_{\mathbf{6}}$ and $\mathbf{E}_{\mathbf{5}}$ occur at $t=10,12$ and 14 , respectively.

We also considered the enhancement of $\mathbf{E}_{\mathbf{1}}$ to the general series of rank 2 simply laced algebras. These configurations are associated to the algebras $\tilde{A}_{2}^{(a)}$ with Dynkin diagram $A=\left(\begin{array}{cc}2 & -a \\ -a & 2\end{array}\right)$, and have trace

$$
\operatorname{Tr} K=4 a^{2}-14 \text {. }
$$

The $a=0$ and $a=1$ configurations with algebras $A_{1} \oplus A_{1}$ and $A_{2}$ have $t=-14$ and $t=-10$, joining the other exotic finite algebras in the hyperbolic classes of negative trace. The case with $a=2$ is just $\widehat{\mathbf{E}}_{\mathbf{1}}$ and has $t=2$ as expected, and thereafter the algebras are strictly hyperbolic and the configurations appear at large positive values of $t$, $a=3$ being at $t=22$. Even more exotic configurations, which we have not explored here, presumably populate the higher- $t$ classes.

We found configurations realizing the parabolic classes with $\left(\begin{array}{cc}1 & -N \\ 0 & 1\end{array}\right)$ for $N \geq-9\left(\widehat{\mathbf{E}}_{\mathbf{N}+\mathbf{9}}\right.$ and $\left.\mathbf{A}_{\mathbf{N}-\mathbf{1}}\right)$ and $\left(\begin{array}{c}-1 N \\ 0-1\end{array}\right)$ for $N \geq-4\left(\mathbf{D}_{\mathbf{N}+\mathbf{4}}\right)$. It is natural to wonder about the remaining classes. One moves down the $\widehat{\mathbf{E}}_{\mathbf{N}}$ and $\mathbf{D}_{\mathbf{N}}$ series by removing $\mathbf{A}$-branes, but at the ends of the series there are no more such branes to remove. However, we can add to the $\widehat{\mathbf{E}}_{\mathbf{0}}$ and $\mathbf{D}_{\mathbf{0}}$ configurations a set of branes with total monodromy 
inverse to that of a single A-brane, namely the configuration with trivial monodromy $\widehat{\mathbf{E}}_{\mathbf{9}}$ with a single $\mathbf{A}$-brane removed, in other words $\widehat{\mathbf{E}}_{\mathbf{8}}=$ $\mathbf{A}^{7} \mathbf{B C B C}$. One can then remove $\mathbf{A}$ branes to continue into the other parabolic classes, adding $\widehat{\mathbf{E}}_{\mathbf{8}}$ factors when necessary. In this fashion we exhaust the parabolic classes. We do not attempt to classify the algebras on these configurations.

As an example consider the $\mathbf{D}_{\mathbf{N}}$ series. $\mathbf{D}_{\mathbf{0}}=\mathbf{B C}$ and has no $\mathbf{A}$ branes which can be removed. As far as the monodromy is concerned $\mathbf{D}_{\mathbf{0}}$ is equivalent to $\widehat{\mathbf{E}}_{\mathbf{9}} \mathbf{D}_{\mathbf{0}}=\mathbf{A}^{8} \mathbf{B C B C B C}$, which has A-branes which can be removed to obtain, for example, $\mathbf{D}_{-1} \equiv \mathrm{A}^{7} \mathbf{B C B C B C}$. Conjugacy classes $\left(\begin{array}{c}-1 N \\ 0-1\end{array}\right)$ for $N<-4$ are thus realized by 7-brane configuration made of $\mathbf{A}$-branes and an odd number of $\mathbf{B C}$ pairs.

In general, an $\widehat{\mathbf{E}}_{\mathbf{9}}$ configuration can be added to any any configuration $\mathbf{G}$ without changing the monodromy:

$$
K(\mathbf{G})=K\left(\mathbf{G} \widehat{\mathbf{E}}_{\mathbf{9}}\right)
$$

and consequently the entire classification possesses a certain $\widehat{\mathbf{E}}_{\mathbf{9}}$-periodicity. To any classified configuration $\mathbf{G}$ with $n$ branes, we can add $k$ factors of $\widehat{\mathbf{E}}_{\mathbf{9}}$ to obtain a new configuration with $n+12 k$ branes and algebra

$$
\mathcal{G}\left(\mathbf{G} \widehat{\mathbf{E}}_{\mathbf{9}} \widehat{\mathbf{E}}_{\mathbf{9}} \cdots \widehat{\mathbf{E}}_{\mathbf{9}}\right) \supseteq \mathcal{G}(\mathbf{G}) \oplus \widehat{E}_{\mathbf{9}} \oplus \cdots \oplus \widehat{E}_{\mathbf{9}} .
$$

In general we have made no attempt to classify these algebras. A few simple cases can be understood; for example $\mathbf{A} \widehat{\mathbf{E}}_{\mathbf{9}}=\widehat{\mathbf{E}}_{\mathbf{1 0}}$. The progression of the $\mathbf{E}_{\mathbf{N}}^{\mathbf{H}}$ series in Table 5 suggests that $\mathbf{E}_{\mathbf{1 0}}^{\mathbf{H}} \cong \mathbf{H}_{\mathbf{0}} \widehat{\mathbf{E}}_{\mathbf{9}}$, and so on.

The algebraic facts that $E_{9}=\widehat{E}_{8}$ and $E_{10}=E_{8}^{H}$ are reflected in equivalence of the corresponding the brane configurations. In Table 6 we depict how beginning with $\mathbf{E}_{\mathbf{8}}$, we can add an A-brane to proceed along the $\mathbf{E}_{\mathbf{N}}$ series, or an $\mathbf{X}_{[\mathbf{3}, \mathbf{1}]}$-brane to move toward loop and then hyperbolic algebras. The table is reflection-symmetric along the diagonal as a result of these equivalences. Because $\mathbf{E}_{\mathbf{9}}^{\mathbf{H}} \cong \widehat{\mathbf{E}}_{\mathbf{1 0}}$, this suggests that we can define the algebra $E_{9}^{H}$ to be equal to $\widehat{E}_{10}$, which is just the loop algebra of $E_{10}$. Notice the central position occupied by the double-loop configuration $\widehat{\mathbf{E}}_{\mathbf{9}}$.

In Table 5 we summarize this discussion, presenting the algebras arising on a collection of 7-branes with total monodromy of trace $|t| \leq 7$. 


\begin{tabular}{|c|c|c|c|c|c|}
\hline$K$-type & $\operatorname{Tr} K$ & $\operatorname{det} A(\mathcal{G}$ & Brane configuration $\mathbf{G}$ & $K$ & $H(t)$ \\
\hline \multirow{5}{*}{ hyp. } & -7 & 9 & $\tilde{\mathbf{E}}_{\mathbf{0}}, \mathbf{H}_{8}$ & $\left(\begin{array}{ll}-7 & 1 \\
-1 & 0\end{array}\right)^{ \pm 1}$ & 2 \\
\hline & -6 & $(2,8), 8$ & $\left(\mathbf{E}_{\mathbf{1}}, \tilde{\mathbf{E}}_{\mathbf{1}}\right), \mathbf{H}_{\mathbf{7}}$ & $\left(\begin{array}{ll}-6 & 1 \\
-1 & 0\end{array}\right)^{ \pm 1}$ & 2 \\
\hline & -5 & 7 & $\mathbf{E}_{2}, \mathbf{H}_{6}$ & $\left(\begin{array}{ll}-5 & 1 \\
-1 & 0\end{array}\right)^{ \pm 1}$ & 2 \\
\hline & -4 & 6 & $\mathbf{E}_{3}, \mathbf{H}_{5}$ & $\left(\begin{array}{ll}-4 & 1 \\
-1 & 0\end{array}\right)^{ \pm 1}$ & 2 \\
\hline & -3 & 5 & $\mathbf{E}_{4}=\mathbf{H}_{4}$ & $\left(\begin{array}{ll}-3 & 1 \\
-1 & 0\end{array}\right)$ & 1 \\
\hline par. & -2 & 4 & $\begin{array}{c}D_{N+4 \geq 0} \\
\left(E_{5}=D_{5}, H_{3}=D_{3}\right)\end{array}$ & $\left(\begin{array}{cc}-1 & N \\
0 & -1\end{array}\right)$ & $\infty$ \\
\hline \multirow{3}{*}{ ell. } & -1 & 3 & $\mathbf{E}_{6}, \mathbf{H}_{2}$ & $-(S T)^{\mp 1}$ & 2 \\
\hline & 0 & 2 & $\mathrm{E}_{\mathbf{7}}, \mathrm{H}_{1}$ & $S^{ \pm 1}$ & 2 \\
\hline & 1 & 1 & $\mathrm{E}_{8}, \mathrm{H}_{\mathbf{0}}$ & $(S T)^{ \pm 1}$ & 2 \\
\hline par. & 2 & $\begin{array}{c}N \\
0\end{array}$ & $\begin{array}{c}\mathbf{A}_{\mathbf{N}-1 \geq 0} \\
\widehat{\tilde{\mathbf{E}}}_{\mathbf{N}+9 \geq 0},\left(\mathbf{E}_{9}=\widehat{\mathbf{E}}_{8}\right)\end{array}$ & $\left(\begin{array}{cc}1 & -N \\
0 & 1\end{array}\right)$ & $\infty$ \\
\hline \multirow{5}{*}{ hyp. } & 3 & -1 & $\mathbf{E}_{10}=\mathbf{E}_{8}^{\mathrm{H}}$ & $\left(\begin{array}{cc}0 & 1 \\
-1 & 3\end{array}\right)$ & 1 \\
\hline & 4 & -2 & $\mathrm{E}_{11}, \mathrm{E}_{7}^{\mathrm{H}}$ & $\left(\begin{array}{cc}0 & 1 \\
-1 & 4\end{array}\right)^{ \pm 1}$ & 2 \\
\hline & 5 & -3 & $\mathrm{E}_{12}, \mathrm{E}_{6}^{\mathrm{H}}$ & $\left(\begin{array}{cc}0 & 1 \\
-1 & 5\end{array}\right)^{ \pm 1}$ & 2 \\
\hline & 6 & -4 & $\mathrm{E}_{13}, \mathrm{E}_{5}^{\mathrm{H}}$ & $\left(\begin{array}{cc}0 & 1 \\
-1 & 6\end{array}\right)^{ \pm 1}$ & 2 \\
\hline & 7 & -5 & $\mathrm{E}_{14}, \mathrm{E}_{4}^{\mathrm{H}}$ & $\left(\begin{array}{cc}0 & 1 \\
-1 & 7\end{array}\right)^{ \pm 1}$ & 2 \\
\hline
\end{tabular}

Table 5: $S L(2, \mathbf{Z})$ conjugacy classes and algebras realized on 7-branes with overall monodromy $K$ (up to conjugation). The upper and lower exponents of the matrices correspond to the first and the second brane configuration, respectively. The brane configurations G, whose notations suggest the algebra $\mathcal{G}$, are defined in the text. The determinant of $A(\mathcal{G})$ is given, except when no algebra is realized, as with $\mathbf{H}_{\mathbf{0}}, \tilde{\mathbf{E}}_{\mathbf{0}}$ and $\mathbf{D}_{\mathbf{0}}$. 


\begin{tabular}{||c|c|c||}
\hline $\mathrm{E}_{8}^{\mathrm{H}}=\mathrm{E}_{8} \mathrm{X}^{2}$ & $\mathrm{E}_{9}^{\mathrm{H}}=\mathrm{AE}_{8} \mathrm{X}^{2}$ & $\mathrm{E}_{10}^{\mathrm{H}}=\mathrm{A}^{2} \mathrm{E}_{8} \mathrm{X}^{2}$ \\
\hline$\widehat{\mathrm{E}}_{8}=\mathrm{E}_{8} \mathrm{X}$ & $\widehat{\mathrm{E}}_{9}=\mathrm{AE}_{8} \mathrm{X}$ & $\widehat{\mathrm{E}}_{10}=\mathrm{A}^{2} \mathrm{E}_{8} \mathrm{X}$ \\
\hline $\mathrm{E}_{8}=\mathrm{A}^{7} \mathrm{BC}^{2}$ & $\mathrm{E}_{9}=\mathrm{AE}_{8}$ & $\mathrm{E}_{10}=\mathrm{A}^{2} \mathrm{E}_{8}$ \\
\hline
\end{tabular}

Table 6: Enhancements of the $\mathbf{E}_{\mathbf{8}}$ brane configuration. In the horizontal direction we add $\mathbf{A}$ branes and in the vertical direction we add $\mathbf{X}=$ $[3,1]$ branes. The brane configurations produce the algebras suggested by their names. This square is reflection symmetric with respect to the diagonal running from the lower left corner to the upper right corner.

\section{Compactifications of type IIB superstrings}

Throughout this paper we have investigated the algebraic properties of the monodromies associated with 7-branes, without considering the physics of the background the 7-branes are realized in. The well-known string theory background that inspired this work is that of IIB strings compactified on $S^{2}$ in the presence of 24 mutually non-local 7-branes. This can also be viewed as a compactification of F-Theory on an elliptic $\mathrm{K} 3$, where the fibration over $\mathbb{P}^{1}$ degenerates at 24 points. In this section we address the question of which algebras actually appear in such a background. Since the finite algebras have already been studied in some detail $[4,19,20]$, our focus here is on the infinite algebras. We find configurations of branes realizing many of the affine and indefinite algebras discussed in this paper, existing in certain regions of the moduli space of compactifications. As emphasized, infinite-dimensional algebras are not associated to singularities, since these brane configurations cannot coalesce (unless the whole K3 degenerates). Thus, the associated theories are always spontaneously broken, and while the massive spectrum does fall into representations of the algebra, states belonging to any particular representation will not in general have the same mass. An additional subtlety is that owing to the global properties of the base, loop junctions around the entire brane configuration can be contracted to a point and are actually trivial.

Another interesting manifold with an elliptic fibration structure is 
the ninth del Pezzo surface $\mathcal{B}_{9}$, also called $\frac{1}{2} \mathrm{~K} 3$ since it is a fibration over $\mathbb{P}^{1}$ degenerating at 12 points. This manifold is not Calabi-Yau and so is not a candidate for F-Theory compactification, but arises as a 4-cycle that can collapse to zero size inside certain Calabi-Yau threefolds. These collapsing 4-cycles lead to interesting theories with tensionless strings in 6D and 5D. D2-branes wrapped on the holomorphic curves of this manifold lead to electrically charged BPS particles in the $\mathcal{N}=2, D=4$ theory in the transverse space of a IIA compactification [21, 22, 23]. The spectra of BPS states can be reproduced by looking at BPS junctions of asymptotic charge $(p, 0)$ on the $\widehat{\mathbf{E}}_{\mathbf{9}}$ configuration. The degree of a holomorphic curve in $\mathcal{B}_{9}$ is then equal to the the $p$-charge of the corresponding junction. The 2-cycles in $\mathcal{B}_{9}$ and their intersection matrix can be determined using the techniques of this paper, even though they do not correspond to junctions of $(p, q)$ strings. The "brane" configuration on this surface will be seen to be $\widehat{\mathbf{E}}_{\mathbf{9}}$, although the global properties of the base will again render certain junctions trivial.

\subsection{Junctions and the homology lattice of $\mathcal{B}_{9}$}

$\mathcal{B}_{9}$ is a complex manifold which can be expressed as an elliptic fibration over the base $B \cong \mathbb{P}^{1}[24,25,26,27]$. The Euler characteristic of $\mathcal{B}_{9}$ is twelve and hence it has twelve degenerate fibers. The positions of the degenerate fibers on the base $B$ are the positions of the twelve "7branes". $\mathcal{B}_{9}$ can also be obtained by blowing up nine generic points on $\mathbb{P}^{2}$. The homology lattice of $\mathcal{B}_{9}$ has as a basis the cycles $l, e_{1}, \ldots, e_{9}$, where $l$ is basic homology class of $\mathbb{P}^{2}$ and $-e_{i}$ are the classes obtained by blowing up the nine points. The intersection numbers of these classes are given by

$$
l^{2}=1, \quad l \cdot e_{i}=0, \quad e_{i} \cdot e_{j}=-\delta_{i j}, \quad \forall i, j \in\{1, \ldots, 9\}
$$


The homology lattice is isomorphic to the lattice $\Gamma^{9,1}=-A\left(E_{8}\right) \oplus \Gamma^{1,1}$, where $\Gamma^{1,1}=\left(\begin{array}{cc}1 & 0 \\ 0 & -1\end{array}\right)$. This can be seen by defining a new basis

$$
\begin{aligned}
\alpha_{i} & =e_{i}-e_{i+1}, \quad 1 \leq i \leq 7, \\
\alpha_{8} & =l-e_{1}-e_{2}-e_{3}, \\
B & =e_{9} \\
F & =3 l-\sum_{i=1}^{9} e_{i} .
\end{aligned}
$$

The classes $B$ and $F$ represent the fiber and the base of the elliptic fibration. In this basis $\alpha_{i}, i=1, \ldots, 8$ have the intersection matrix $-A\left(E_{8}\right) . B+F$ and $B$ generate the basis of the lattice $\Gamma^{1,1}$ and have the intersection matrix $\left(\begin{array}{cc}1 & 0 \\ 0 & -1\end{array}\right)$. It is not possible to get the hyperbolic lattice $\left(\begin{array}{ll}0 & 1 \\ 1 & 0\end{array}\right)$ by a change of basis which preserves the fact that the basis should be integral homology classes, and therefore it follows that the ten dimensional homology lattice of $\mathcal{B}_{9}$ is not the root lattice of $E_{10}$.

Since the base of the elliptic fibration of $\mathcal{B}_{9}$ is compact, the total monodromy of the "7-brane" configuration must be unity, since the branch cuts have nowhere to go. Since there are twelve branes, the configuration in question must be $\widehat{\mathbf{E}}_{\mathbf{9}}$. The full junction lattice on $\widehat{\mathbf{E}}_{\mathbf{9}}$ is twelve-dimensional. The condition of vanishing asymptotic charge is equivalent, on this compact surface, to the condition of zero boundaries on the curves. The remaining ten-dimensional lattice is further reduced by the fact that since the 7-branes are on a sphere, any loop around the entire configuration can be shrunk to a point. This means that both imaginary root junctions must be set equal to the zero junction:

$$
\delta_{1}=\delta_{2}=0 .
$$

The remaining eight-dimensional lattice is just $-A\left(E_{8}\right) \cong \subset \Gamma^{9,1}$, corresponding to the $A\left(E_{8}\right)$ part of the $\mathcal{B}_{9}$ junction lattice. Since the junctions always have one dimension in each the base and the fiber, the cycles corresponding to wrapping completely either the base or the fiber are invisible in the junction picture.

\subsection{Junctions and the homology lattice of $\mathrm{K3}$}

The second homology group of K3 is a 22-dimensional lattice of signature $(19,3): H^{2}(\mathrm{~K} 3, \mathbf{Z})=\Gamma^{19,3}$. In a particular basis the intersection 
matrix is given by

$$
\left[-A\left(E_{8}\right)\right] \oplus\left[-A\left(E_{8}\right)\right] \oplus H \oplus H \oplus H
$$

where $A\left(E_{8}\right)$ is the Cartan matrix of $E_{8}$ and $H=\left(\begin{array}{l}01 \\ 10\end{array}\right)$. Out of the three $H$-factors, one is the intersection matrix of $[F]$ and $[B]+[F]$, where $[B]$ is the homology class of the base and $[F]$ is the homology class of the fiber.

Even though we have twenty four branes on the two-sphere associated to the K3, a junction now cannot have asymptotic charge and therefore the junction lattice is at most twenty two dimensional. In addition, since the total monodromy around the entire configuration is trivial there are two linearly independent junctions encircling all the branes. These junctions can be shrunk to a point on the other side of the base $B \cong \mathbb{P}^{1}$ and are therefore trivial. As a consequence, the lattice of junctions is twenty dimensional. It corresponds to the lattice $\Gamma^{18,2}=\left[-A\left(E_{8}\right)\right] \oplus\left[-A\left(E_{8}\right)\right] \oplus H \oplus H$, the sub-lattice of $H^{2}(\mathrm{~K} 3, \mathbf{Z})$ generated by the classes other than $[B]$ and $[F]$. Fig. 8 shows a basis of this sub-lattice, with two $\widehat{\mathbf{E}}_{\mathbf{9}}$ brane configurations, similar to a basis used in [28]. (We explain in the next section how $\widehat{\mathbf{E}}_{\mathbf{9}} \widehat{\mathbf{E}}_{\mathbf{9}}$ arises on the full K3.) The part of the basis giving one of the $H$ 's consists of $\delta_{3,1}$ and $\delta_{3,1}+A A$ where $\delta_{3,1}$ is the $(3,1)$-loop and $A A$ stands for the string connecting the A-branes. Similarly $\delta_{1,0}$ and $\delta_{1,0}+X X$ generate the other $H$ and the root junctions of the two $E_{8}$ 's give $\left[-A\left(E_{8}\right)\right] \oplus\left[-A\left(E_{8}\right)\right]$.

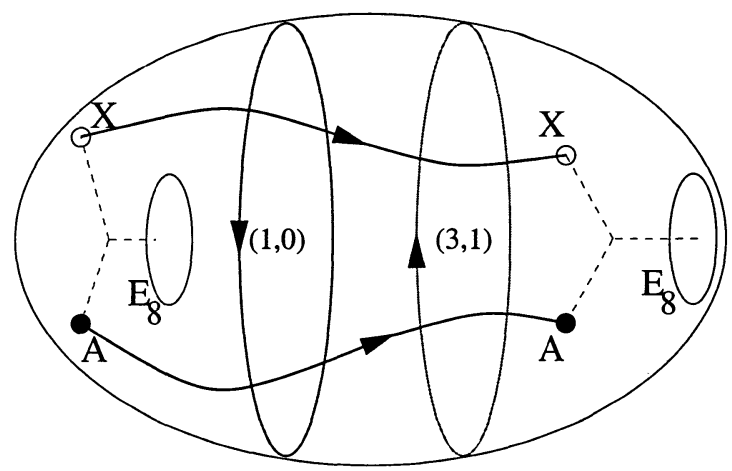

Figure 8: The 24 7-branes of the full $\mathrm{K} 3$ are grouped to uncover the $\Gamma^{18,2}=\left[-A\left(E_{8}\right)\right] \oplus\left[-A\left(E_{8}\right)\right] \oplus H \oplus H$ part of the homology lattice. 


\subsection{Brane configurations on the full $\mathrm{K} 3$}

Let us now examine configurations realizing infinite-dimensional algebras on $\mathrm{K} 3$. We can start with eight $\mathbf{H}_{\mathbf{1}}$ singularities:

$$
\mathrm{K3}=(\mathbf{A A C})(\mathbf{A A C})(\mathbf{A A C})(\mathbf{A A C})(\mathrm{AAC})(\mathbf{A A C})(\mathbf{A A C})(\mathbf{A A C})
$$

The total monodromy of this configuration is unity, as it should be. Since the branch cuts all go downwards to a single point on the two sphere and there are no more branes around, branes can move cyclically. We now recall that $\mathbf{C A A}=\mathbf{A A B}$. This allows one to write [4]

$$
\mathrm{AACAAC}=\mathrm{AAAABC}=\mathrm{D}_{4} .
$$

Thus the full configuration is equivalent to

$$
\mathrm{K} 3 \cong\left(\mathrm{A}^{4} \mathrm{BC}\right)\left(\mathrm{A}^{4} \mathrm{BC}\right)\left(\mathrm{A}^{4} \mathrm{BC}\right)\left(\mathrm{A}^{4} \mathrm{BC}\right)=\mathrm{D}_{4} \mathbf{D}_{4} \mathrm{D}_{4} \mathrm{D}_{4},
$$

the familiar configuration giving rise to an $s o(8)^{4}$ algebra [29]. The algebra on this configuration is even larger when we take into account junctions that stretch between $\mathbf{D}_{\mathbf{4}}$ factors. We can now combine them in pairs and find

$$
\mathbf{K} 3 \cong\left(\mathrm{A}^{8} \mathrm{BC} \mathrm{BC}\right)\left(\mathrm{A}^{8} \mathrm{BC} \mathrm{BC}\right)=\widehat{\mathbf{E}}_{\mathbf{9}} \widehat{\mathbf{E}}_{\mathbf{9}} .
$$

Let us, however impose global constraints. Each $\widehat{\mathbf{E}}_{\mathbf{9}}$ has a pair of associated $\boldsymbol{\delta}$ junctions, one each of $(1,0)$ and $(3,1)$ charges: $\boldsymbol{\delta}_{\mathbf{A}}^{i}, \boldsymbol{\delta}_{\mathbf{X}}^{i}$. In fact $\boldsymbol{\delta}_{\mathbf{A}}^{1}+\boldsymbol{\delta}_{\mathbf{A}}^{2}$ and $\boldsymbol{\delta}_{\mathbf{X}}^{1}+\delta_{\mathbf{X}}^{2}$ are the two junctions that wind around the entire configuration, and hence are equivalent to zero. We must impose a condition on the basis strings,

$$
\delta_{\mathbf{A}}^{1}=-\delta_{\mathbf{A}}^{2}, \quad \delta_{\mathbf{X}}^{1}=-\delta_{\mathbf{X}}^{2} .
$$

Thus instead of four nontrivial imaginary roots on the total algebra, we have just two, which we can think of as the loops "caught" in between the two brane configurations, as in Fig. 8. Therefore, an arbitrary junction will be characterized by two levels only. The subalgebra readily identified here is therefore the quotient $\left(\widehat{E}_{9} \oplus \widehat{E}_{9}\right) /\left(K_{A}^{1}+K_{A}^{2}, K_{X}^{1}+K_{X}^{2}\right)$, where $\left(K_{A}^{1}, K_{X}^{1}\right)$ and $\left(K_{A}^{2}, K_{X}^{2}\right)$ are the central elements of the first and second $\widehat{E}_{9}$ respectively. Note that a weight vector of this algebra is defined by $18(=10 \times 2-2)$ integers and thus it cannot capture the full set of invariant charges (20 of them) of the associated junction. 
Alternatively, we can associate one imaginary root in Fig. 8 to one of the $E_{8}$ 's and the other root to the other $E_{8}$ obtaining an equivalent description in terms of an $E_{9} \oplus E_{9}$ subalgebra without a quotient. In this case the failure to describe the full junction lattice can be ascribed to the existence of two nontrivial junctions that are $E_{9} \oplus E_{9}$ singlets. Indeed, consider the side with brane configuration $\mathbf{A} \widehat{\mathbf{E}}_{\mathbf{8}}=\mathbf{A E}_{\mathbf{8}} \mathbf{X}_{[\mathbf{3}, \mathbf{1}]}$. The relevant singlet here is tadpole like, with the loop surrounding the $\widehat{\mathbf{E}}_{\mathbf{8}}$ (carrying one $E_{9}$ ) and the leg a $(1,0)$-string ending on the A-brane (recall the discussion around (3.25)). Exactly analogous considerations apply to the other side.

The $\mathbf{E}_{\mathbf{1 0}} \oplus \mathbf{E}_{\mathbf{1 0}}$ configuration. It is natural to rearrange the branes so that it is the junctions stretching in between the two configurations which are removed by the global constraints. Rewrite the configuration $\mathbf{K 3}$ in (7.8) as

$$
\begin{aligned}
\mathrm{K} 3 & \cong\left(\mathbf{A}^{8} \mathbf{B C C X}_{[\mathbf{3}, \mathbf{1}]}\right)\left(\mathrm{A}^{8} \mathbf{B C C X}_{[\mathbf{3}, \mathbf{1}]}\right) \\
& =\left(\mathrm{AE}_{\mathbf{8}} \mathbf{X}_{[\mathbf{3}, \mathbf{1}]}\right)\left(\mathbf{A E}_{\mathbf{8}} \mathbf{X}_{[\mathbf{3}, \mathbf{1}]}\right),
\end{aligned}
$$

where each $\left(\mathbf{A E}_{\mathbf{8}} \mathbf{X}_{[\mathbf{3}, \mathbf{1}]}\right)$ has unit monodromy and so all its branch cuts meet at a point. Since $\mathbf{A E}_{\mathbf{8}} \mathbf{X}_{[\mathbf{3}, \mathbf{1}]}=\mathbf{X}_{[\mathbf{3}, \mathbf{1}]} \mathbf{A E}_{\mathbf{8}}$ we can move one $\mathbf{X}_{[\mathbf{3}, \mathbf{1}]}$ brane around the other configuration entirely, and regroup the branes:

$$
\mathrm{K} 3 \cong\left(\mathrm{AE}_{\mathbf{8}} \mathrm{A}\right)\left(\mathrm{E}_{8} \mathbf{X}_{[3,1]} \mathbf{X}_{[3,1]}\right)=\mathbf{E}_{10} \mathbf{E}_{10}^{\prime}
$$

The brane configurations $\mathbf{A E}_{\mathbf{8}} \mathbf{A}$ and $\mathbf{E}_{\mathbf{8}} \mathbf{X}_{[\mathbf{3 , 1}]} \mathbf{X}_{[\mathbf{3}, \mathbf{1}]}$ are both equivalent to $\mathbf{E}_{\mathbf{1 0}}$. Therefore both configurations realize the algebra $E_{10}$. They have inverse monodromies, and there is a branch cut stretching between them. In $S L(2, \mathbf{Z})$ a matrix and its inverse have the same trace; since there is a single conjugacy class at trace equal $(+3)$, the second configuration in fact must be conjugate (and equivalent) to the first.

An interesting phenomenon of junction localization occurs for the configuration (7.11). Since $E_{10}$ has no conjugacy classes a singlet can be realized on $\mathbf{E}_{10}$ with any asymptotic charge. A singlet of $E_{10} \oplus E_{10}$ will then be a loop around the first $\mathbf{E}_{\mathbf{1 0}}$ with some asymptotic charge $(p, q)$ and another loop around the second $\mathbf{E}_{\mathbf{1 0}}$ configuration with asymptotic charge $(-p,-q)$, as shown in Fig. 9(a). From the way singlets are constructed [6] it is clear that this singlet can be realized as shown in Fig. 9(b) and is thus zero in homology because it can be contracted to a point on the other side of the sphere. Consider a generic junction 
$\mathbf{J}$ stretching between the two $\mathbf{E}_{\mathbf{1 0}}$ configurations taking an asymptotic charge $(p, q)$ from one $\mathbf{E}_{\mathbf{1 0}}$ to the other $\mathbf{E}_{\mathbf{1 0}}^{\prime}$. By adding a suitable singlet we can eliminate the string going between the configurations. Thus any junction $\mathbf{J}$ is equivalent to the sum of two junctions $\mathbf{J}_{1}$ and $\mathbf{J}_{2}$ localized on the first and the second configuration respectively.

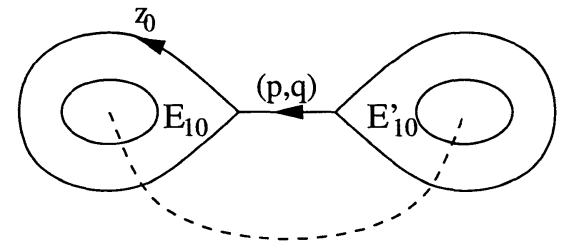

(a)

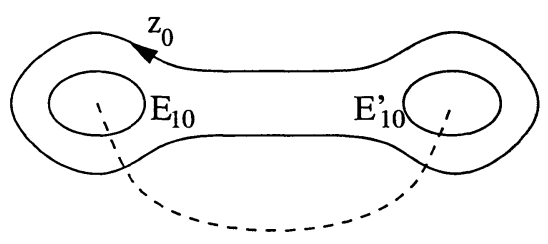

(b)

Figure 9: (a) A junction which is a singlet under both $E_{10}$ 's. (b) The same singlet junction is now seen to be trivial on a compact surface.

The full spectrum of junctions, however is not just those that are in the adjoint of $E_{10} \oplus E_{10}$. Although any junction can be expressed as $\mathbf{J}=\mathbf{J}_{1}+\mathbf{J}_{2}$ on the two $\mathbf{E}_{\mathbf{1 0}}$ 's, the only requirement for it to be BPS is that $\mathbf{J}^{2} \geq-2$, and the existence of a BPS junction is then guaranteed by the geometry of $\mathrm{K} 3[30,31]$ with a connected holomorphic surface for the corresponding 2-cycle [30]. The two pieces need not both satisfy $\mathbf{J}_{1}^{2} \geq-2$ and $\mathbf{J}_{2}^{2} \geq-2$, and so one of them need not correspond to a root of $E_{10}$, but to some other weight vector.

Both the $E_{9} \oplus E_{9}$ and the $E_{10} \oplus E_{10}$ configurations nicely capture aspects of the homology lattice of $\mathrm{K} 3$. The $E_{10} \oplus E_{10}$ configuration, however, is complete since there is a one to one correspondence between the twenty Dynkin labels of a weight vector and the twenty invariant charges of an arbitrary junction. On the other hand the $E_{9} \oplus E_{9}$ lattice is only eighteen dimensional and junctions cannot be reconstructed from the weight vectors alone.

Other configurations. If we consider asymmetric configurations one can get even larger algebras. It is well-known that no more than 18 branes can be made mutually local, corresponding to the fact that the elliptic fibration of $\mathrm{K} 3$ is determined by 18 parameters [1]. To see such a configuration, begin with (7.8) and apply a global $S L(2, \mathbf{Z})$ transformation with the A-brane monodromy $K_{A}$ to convert into the 
$\tilde{\mathbf{E}}_{\mathbf{N}}$ presentation:

$$
\begin{aligned}
& \left(\mathrm{A}^{8} \mathrm{BCCX}_{[3,1]}\right)\left(\mathrm{A}^{8} \mathrm{BCC} \mathrm{X}_{[3,1]}\right) \\
& \rightarrow\left(\mathrm{A}^{9} \mathbf{X}_{[2,-1]} \mathbf{C X}_{[4,1]}\right)\left(\mathbf{A}^{9} \mathbf{X}_{[2,-1]} \mathbf{C X}_{[4,1]}\right) \\
& =A^{18} X_{[2,-1]} C X_{[4,1]} X_{[2,-1]} C X_{[4,1]} \\
& =\mathbf{A}_{17} \widehat{\mathbf{E}}_{\mathbf{0}} \widehat{\mathbf{E}}_{\mathbf{0}} \text {, }
\end{aligned}
$$

The identification of the $\mathbf{A}_{\mathbf{1 7}} \widehat{\mathbf{E}}_{\mathbf{0}} \widehat{\mathbf{E}}_{\mathbf{0}}$ combination is consistent with this algebra arising in [32]. The middle configuration in (7.12) can be turned into:

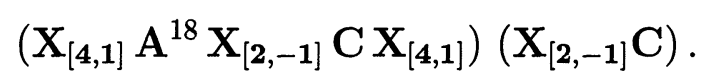

The left-hand piece can be thought of as " $\mathbf{E}_{\mathbf{1 8}}^{\mathbf{H}}$ ", while the right-hand piece is just $\mathbf{E}_{\mathbf{0}}$.

\subsection{Metrics associated to 7-branes}

Some intuition can be gained by examining the metrics on the two sphere. First we claim that any $\mathbf{D}_{\mathbf{N}}$ configuration leads to a conical geometry with deficit angle $\pi$. This is certainly well-known for the $\mathbf{D}_{\mathbf{4}}$ case, and is proven as follows. We recall [33] that the metric on the two sphere is of the form $d s_{p, q}=\left|h_{p, q}(z) d z\right|$ where

$$
h_{p, q}(z)=(p-q \tau) \eta^{2}(\tau) \prod_{i}\left(z-z_{i}\right)^{-1 / 12} .
$$

We consider the bunch of branes centered around $z=0$, close to each other so that for sufficiently large $z$ we can take all $z_{i}$ in the above expression to be zero. The branch cuts go downwards from $z=0$. The monodromy of the configuration is used to get an expression for $\tau(z)$ and then plugged back into (7.14) to get the $z$-behavior of $h_{p, q}$. We will consider the metric $d s_{1,0}$ as seen by $(1,0)$ strings. The monodromy $K$ of the $\mathbf{D}_{\mathbf{N}}$ configuration indicated in the main table implies that $\tau_{R}=\tau_{L}-(N-4)$, where $\tau_{R}, \tau_{L}$ denote the values of $\tau$ immediately to the right and immediately to the left of the cut, respectively. Such monodromy requires the behavior

$$
\tau(z) \sim \frac{1}{2 \pi i}(N-4) \ln z, \quad-\pi / 2<\arg z<3 \pi / 2 .
$$


Note moreover that as $z \rightarrow 0$, we find $\operatorname{Im} \tau \rightarrow i \infty \cdot(N-4)$ which is consistent if $N \geq 4$, but inconsistent if $N<4$. This, of course, only means that we cannot get a genuine singularity for $N<4$, as we have discussed repeatedly. For $\tau \rightarrow i \infty$ we also have $\eta^{2}(\tau) \sim \exp (2 \pi i \tau / 12)$. Thus, for $N \geq 4$, and given that we have a total of $(N+2)$ branes, we get

$$
h_{1,0}(z) \sim z^{\frac{N-4}{12}} z^{-\frac{N+2}{12}} \sim z^{-1 / 2} .
$$

This behavior indicates that away from the singularity we have a flat conical geometry with defect angle of $\pi$, as claimed.

The computations are rather similar for the affine exceptional series $\widehat{\mathbf{E}}_{\mathrm{N}}$. The relevant monodromy $K$ indicated in the main table implies that $\tau_{R}=\tau_{L}+(9-N)$, which requires

$$
\tau(z) \sim-\frac{1}{2 \pi i}(9-N) \ln z, \quad-\pi / 2<\arg z<3 \pi / 2 .
$$

Note that for the range of interest $N \leq 9$ as $|z| \rightarrow 0$, we find Im $\tau \rightarrow-i \infty$ indicating again that the branes cannot be brought together. On the other hand, for $|z| \rightarrow \infty$ we get $\tau \rightarrow+i \infty$ and the behavior of the metric far away from the configuration can be discussed. Since the number of branes is $(N+3)$ this time we get

$$
h_{1,0}(z) \sim z^{-\frac{(9-N)}{12}} z^{-\frac{N+3}{12}} \sim 1 / z .
$$

The metric $d s_{1,0}=|d z / z|$ away from any $\widehat{\mathbf{E}}_{\mathbf{N}}$ configuration is that of a flat cylinder; effectively we have a deficit angle of $2 \pi$. This is in accord with the expectation of the particular case when two nearby $\mathbf{D}_{\mathbf{4}}$ configurations are thought as a $\widehat{\mathbf{E}}_{\mathbf{9}}$ configuration. The defect angle of $\pi$ carried by each $\mathbf{D}_{\mathbf{4}}$ adds up to a total defect angle of $2 \pi$ for the complete $\widehat{\mathbf{E}}_{\mathbf{9}}$ configuration. Two affine configurations carry the necessary defect angle to produce a two sphere.

For configurations leading to Lorentzian algebras the nature of the metric is not easily elucidated. For example the $\mathbf{E}_{\mathbf{1 0}}$ configuration has a monodromy conjugate to $\left(\begin{array}{cc}0 & 1 \\ -1 & 3\end{array}\right)$, implying that $\tau \rightarrow 1 /(3-\tau)$. The fact that the fixed points of this transformation are irrational numbers on the real line-points that are not conjugate to points in the modular domain - indicates that nowhere in the sphere can the metric be expected to be simple. An understanding of metrics on the sphere for the case of the double $\mathbf{E}_{10}$ configuration would be of interest. 


\section{Conclusion and Open Questions}

We presented a systematic survey of the spectrum generating algebras arising on 7-brane configurations. Relaxing the condition that the branes be collapsible to a singularity of $\mathrm{K} 3$, a wide range of algebras were found, including semisimple, affine and indefinite Kac-Moody algebras as well as more exotic ones. We showed that on the 24 7-branes of the full $\mathrm{K} 3$ a $E_{10} \oplus E_{10}$ algebra arises naturally.

Many questions are still open. We used three $S L(2, \mathbf{Z})$ invariants (conjugacy class of the overall monodromy, number of branes and constraints on the asymptotic charges) to classify the equivalent configurations and thus the algebras.. These were sufficient for all the configurations we have examined but there remains the possibility that other invariants are necessary to uniquely identify the resulting algebra. We also observe that not every simply laced Lie algebra is realized, for example, only the algebras in the exceptional series admit affine extensions.

Although the root lattice of the $E_{10} \oplus E_{10}$ algebra can be identified with the homology of the junctions on $\mathrm{K} 3$, the algebra generated by the complete set of BPS junctions is larger than $E_{10} \oplus E_{10}$. It would be interesting to identify the algebra for which the full set of BPS junctions is the adjoint representation. The Narain lattice arising from compactification on a two torus of the heterotic string is also equivalent to the root lattice of $E_{10} \oplus E_{10}$. This being the heterotic dual to the IIB compactification we have examined, it may be possible to relate explicitly the junction lattice to the Narain lattice.

The algebras on the 7-branes should constrain and organize the BPS spectrum of the corresponding D3 brane probe theories. We believe that the affine and indefinite algebras studied here will prove useful in studying the spectrum of the field theories on D3 brane probes.

\section{Acknowledgments}

We are grateful to $\mathrm{V}$. Kac for invaluable help in identifying various infinite dimensional algebras. Thanks are due to A. Hanany, C. Vafa, E. Witten and D. Zagier for their useful comments and questions and 
to R. Borcherds for very helpful correspondence.

This work was supported by the U.S. Department of Energy under contract \#DE-FC02-94ER40818.

\section{A Appendix: Binary quadratic forms and ideal classes of quadratic fields}

In this appendix we elaborate on the relation between binary quadratic forms and ideal classes in quadratic fields and explain how to calculate the number of conjugacy classes of binary quadratic forms of given discriminant $\widehat{d}$. For more details see [34, 35, 36]

Algebraic number theory: A number $\theta$ is called an algebraic number if it is a root of a polynomial with coefficients in $\mathbb{Q}$ and it is called an algebraic integer if it is a root of a polynomial whose leading coefficient is 1 and all the others are integers. By adjoining an algebraic number $\theta$ to the rational field, one obtains an extension of $\mathbb{Q}$, which is also a field and is denoted by $\mathbb{Q}(\theta)$. The set of all algebraic integers of $\mathbb{Q}(\theta)$ forms a ring, $\mathcal{O}(\theta)$ which has many properties similar to those of the ring of usual integers, for example one can define the notion of a prime number in an exactly analogous way.

A subset $I \subset \mathcal{O}(\theta)$ is an ideal in $\mathbb{Q}(\theta)$ if $\alpha, \beta \in I$ and $\lambda, \mu \in \mathcal{O}(\theta)$ implies $\lambda \alpha+\mu \beta \in I$ and it is called a prime ideal if $\alpha, \beta \in \mathcal{O}(\theta), \alpha \beta \in I$ implies that either $\alpha \in I$ or $\beta \in I$. An amazing fact is that in any ring of integers every ideal can be uniquely factorized into prime ideals. When $I$ is the set of all multiples of some element of $\mathcal{O}(\theta)$ then we call it principal and a ring of integers is called a principal ideal domain if all its ideals are principal. (The ring of ordinary integers has this property: any ideal of $\mathbf{Z}$ is the set of all multiples of a given integer n.) Principal ideal domains have the property that each integer can be uniquely factorized in terms of prime integers, thus these are unique factorization domains as well.

In a generic ring the non-uniqueness of factorization is characterized by the number of non-principal ideals as follows. One can extend the set of ideals of $\mathcal{O}(\theta)$ to a multiplicative group, $I(\theta)$ by adding the so 
called fractional ideals of $\mathbb{Q}(\theta)$ to it. The set of principal ideals $P(\theta)$ form a subgroup of $I(\theta)$. The quotient group $C(\theta)=I(\theta) / P(\theta)$ is called the class group of field $\mathbb{Q}(\theta)$ and its order is the class number of the algebraic extension. A fundamental theorem of algebraic number theory is that for any finite algebraic extension of the rational numbers, the class group is always a finite group.

Quadratic fields: For $\theta$ satisfying $\theta^{2}-D=0$ with $D$ a square free integer, $\mathbb{Q}(\theta)$ is called a quadratic field. The quadratic field is called real or imaginary when $D>0$ or $D<0$, respectively. A generic element of $\mathbb{Q}(\sqrt{D})$ can be written as

$$
\alpha=x+y \sqrt{D}, \quad x, y \in \mathbb{Q},
$$

while its conjugate $\alpha^{\prime}$, norm $N(\alpha)$ and trace $S(\alpha)$ are defined as:

$$
\alpha^{\prime}=x-y \sqrt{D}, \quad N(\alpha)=\alpha \cdot \alpha^{\prime}, \quad S(\alpha)=\alpha+\alpha^{\prime} .
$$

When $N(\alpha), S(\alpha) \in \mathbf{Z}$ then $\alpha \in \mathcal{O}(\sqrt{D})$ thus the following holds for the set of algebraic integers $\mathcal{O}(\sqrt{D}) \subset \mathbb{Q}(\sqrt{D})$ :

- If $D=2,3(\bmod 4)$ then $\{1, \sqrt{D}\}$ is a basis of $\mathcal{O}(\sqrt{D})$ with discriminant $\widehat{d}=\left|\begin{array}{cc}1 & \sqrt{D} \\ 1 & -\sqrt{D}\end{array}\right|^{2}=4 D \bullet$ If $D=1(\bmod 4)$ then $\left\{1, \frac{1+\sqrt{D}}{2}\right\}$ is a basis of $\mathcal{O}(\sqrt{D})$ with discriminant $\widehat{d}=\left|\begin{array}{cc}1 & \frac{1+\sqrt{D}}{2} \\ 1 & \frac{1-\sqrt{D}}{2}\end{array}\right|^{2}=D$. In both cases the basis of algebraic integers is given by $\left\{1, \frac{\widehat{d}+\sqrt{\hat{d}}}{2}\right\}$, where $\widehat{d}$ is the discriminant of the field $\mathbb{Q}(\sqrt{D})$.

Two ideals $M$ and $P \subset \mathcal{O}(\sqrt{D})$ are equivalent $(M \sim P)$ if $\exists \lambda \in$ $\mathbb{Q}(\sqrt{D})$ such that $M=\lambda \cdot P$ and they are strictly equivalent $(M \approx P)$ if $N(\lambda)>0$. For imaginary fields equivalence implies strict equivalence because $N(\lambda)>0, \forall \lambda \in \mathbb{Q}(\sqrt{D})$, while in the real case $M \sim P$ implies either $M \approx P$ or $M \approx \sqrt{\hat{d}} P$ as $N(1)>0$ and $N(\sqrt{D})<0$ and thus an equivalence class of ideals splits into at most two strict equivalence classes. Let us denote with $h(\widehat{d})$ and $h_{0}(\widehat{d})$ the number of equivalence classes and that of strict equivalence classes, respectively, of the quadratic field $\mathbb{Q}(\sqrt{D})$ with discriminant $\widehat{d}$. Then $h_{0}(\widehat{d}) \leq 2 h(\widehat{d})$. Moreover, equivalence implies strict equivalence exactly when $(\sqrt{D}) \approx(1)$ which we can write as $\sqrt{D} \epsilon=\lambda$ for some unit $\epsilon$. For $D>0, N(\lambda)>0$ only when $N(\epsilon)=-1$ which implies that the fundamental unit satisfies 
$N\left(\epsilon_{F}\right)=-1$. Thus for a square free integer $D$ we have the following result:

$$
h_{0}(\widehat{d})=\left\{\begin{aligned}
h(\widehat{d}), & \text { if } D<0 \\
h(\widehat{d}), & \text { if } D>0 \text { and } N\left(\epsilon_{F}\right)=-1 \\
2 h(\widehat{d}), & \text { if } D>0 \text { and } N\left(\epsilon_{F}\right)=+1
\end{aligned}\right.
$$

Ideals and binary quadratic forms: Let $M=(\alpha, \beta)$ be an ideal in $\mathbb{Q}(\sqrt{D})$ with basis $\{\alpha, \beta\}$ then we associate with it the following binary quadratic form:

$$
\begin{aligned}
& Q_{M}(x, y)=(\alpha x+\beta y)\left(\alpha^{\prime} x+\beta^{\prime} y\right) / q \\
& \text { where } q=\operatorname{gcd}\left(\alpha \alpha^{\prime}, \alpha \beta^{\prime}+\beta \alpha^{\prime}, \beta \beta^{\prime}\right) .
\end{aligned}
$$

If $P \approx M$ and $\{\bar{\alpha}, \bar{\beta}\}$ is a basis of $P$ then $\{\lambda \bar{\alpha}, \lambda \bar{\beta}\}$ is a basis of $M$ for some $\lambda$ of positive norm and there exists a matrix $K$ of determinant +1 such that

$$
\left(\begin{array}{l}
\lambda \bar{\alpha} \\
\lambda \bar{\beta}
\end{array}\right)=K\left(\begin{array}{l}
\alpha \\
\beta
\end{array}\right)
$$

Thus the quadratic forms associated with the two ideals are related by an $S L(2, \mathbf{Z})$ transformation and thus are equivalent. The values that this quadratic form takes are exactly the norms of the integral ideals in the corresponding equivalence class of ideals.

Conversely, given a quadratic form $Q(x, y)=A x^{2}+B x y+C y^{2}$, we can associate the following ideal $M(Q)$ with it,

$$
M(Q)=(\alpha, \beta)=\left\{\begin{array}{lll}
\left(A, \frac{B+\sqrt{\hat{d}}}{2}\right) & \text { if } & A>0 \\
\left(A, \frac{B+\sqrt{\hat{d}}}{2}\right) \sqrt{\hat{d}} & \text { if } & A<0
\end{array}\right\}, \quad \widehat{d}=B^{2}-4 A C .
$$

Under a transformation by an $S L(2, \mathbf{Z})$ matrix $K=\left(\begin{array}{ll}a & b \\ c & d\end{array}\right)$ the quadratic form becomes $Q^{\prime}(x, y)=A^{\prime} x^{2}+B^{\prime} x y+C^{\prime} y^{2}$ where

$$
\left(\begin{array}{l}
A^{\prime} \\
B^{\prime} \\
C^{\prime}
\end{array}\right)=\left(\begin{array}{ccc}
a^{2} & a c & c^{2} \\
2 a b & a d+b c & 2 c d \\
b^{2} & b d & d^{2}
\end{array}\right)\left(\begin{array}{l}
A \\
B \\
C
\end{array}\right) \equiv S\left(\begin{array}{l}
A \\
B \\
C
\end{array}\right)
$$


The matrix $S$ belongs to $S L(3, \mathbf{Z})$ and $\operatorname{Tr} S=(\operatorname{Tr} K)^{2}-1$. The basis of the ideal associated with the quadratic form $Q$ undergoes an $S L(2, \mathbf{Z})$ transformation as well as scaling by a positive norm number. Since ideals whose basis are related by an $S L(2, \mathbf{Z})$ transformation and an overall scaling are (strictly) equivalent we see that ideals associated with equivalent quadratic forms are strictly equivalent. If we denote by $h_{+}(\widehat{d})$ the number of equivalence classes of binary quadratic forms with discriminant $\widehat{d}$, then

$$
h_{+}(\widehat{d})=h_{0}(\widehat{d})
$$

Jacobi symbol: Let the prime factorization of $a$ be $a=\prod_{i} p_{i}^{c_{i}}$ and let $p$ be an odd prime, then we define $(a / p) \equiv \prod_{i}\left(p_{i} / p\right)^{c_{i}}$ where

$$
\left(p_{i} / p\right) \equiv\left\{\begin{array}{rll}
0, & \text { if } & p_{i} \equiv 0(\bmod p) \\
+1, & \text { if } x^{2} \equiv p_{i}(\bmod p) \text { is solvable } \\
-1, & \text { if } x^{2} \equiv p_{i}(\bmod p) \text { is unsolvable }
\end{array}\right.
$$

while for $p=2$ we define

$$
(a / 2)=\left\{\begin{aligned}
0, & \text { if } a \text { is even } \\
+1, & \text { if } a \equiv 1(\bmod 8) \\
-1, & \text { if } a \equiv 5(\bmod 8)
\end{aligned}\right.
$$

Theorem ([37]). If $\widehat{d}=f^{2} d>0$ is such that $d$ is the discriminant of some quadratic field then

$$
\begin{aligned}
h_{+}\left(f^{2} d\right) & =h_{0}\left(f^{2} d\right) \\
& =\left\{\begin{array}{c}
h(d) f \prod_{p \mid f}\left(1-\frac{(d / p)}{p}\right) \cdot \frac{1}{E}, \\
2 h(d) f \prod_{p \mid f}\left(1-\frac{(d / p)}{p}\right) \cdot \frac{1}{E},
\end{array} \quad \text { if } \quad N\left(\epsilon_{f}\right)=-1,\right.
\end{aligned}
$$

In the above equation $\epsilon_{f}$ is the fundamental unit of $\mathcal{O}_{f}(\sqrt{D})$, the ring of integers generated by $\left\{1, f\left(\frac{d+\sqrt{d}}{2}\right)\right\}$. $E$ is the least positive integer such that $\epsilon_{F}^{E}=\epsilon_{f}$, where $\epsilon_{F}$ is the fundamental unit of $\mathcal{O}(\sqrt{D})$, the ring of integers of $\mathbb{Q}(\sqrt{D})$ and $p$ is a prime divisor of $f$.

We now illustrate the use of this theorem on a concrete example. To this end let us calculate the number of conjugacy classes in $S L(2, \mathbf{Z})$ 
with trace $t=6$. The discriminant is $\widehat{d}=t^{2}-4=2^{5}$ which implies $f=2$ and $d=4 D=8$. Thus the quadratic field we are considering is $\mathbb{Q}(\sqrt{2})$. The fundamental unit of the ring of integers $\mathcal{O}(\sqrt{2})$ is $\epsilon_{F}=$ $1+\sqrt{2}$ while that of the subring $\mathcal{O}_{2}(\sqrt{2})$ is $\epsilon_{f}=3+2 \sqrt{2}=\epsilon_{F}^{2}$ which implies $E=2$ and $N\left(\epsilon_{f}\right)=+1$. The class number $h(2)$ of $\mathbb{Q}(\sqrt{2})$ is 1 [36] and direct substitution into (A.11) yields $h_{+}\left(2^{5}\right)=2$. We list a few more examples in the following table.

\begin{tabular}{|c|c|c|c|c|c|c|c|c|c|c|}
\hline$t$ & $\hat{d}=d f^{2}$ & $f$ & $d$ & $\mathbb{Q}(\sqrt{D})$ & $\epsilon_{F}$ & $\epsilon_{f}$ & $N\left(\epsilon_{f}\right)$ & $E$ & $h(D)$ & $h_{+}(\widehat{d})$ \\
\hline \hline 3 & 5 & 1 & 5 & $\mathbb{Q}(\sqrt{5})$ & $\frac{1+\sqrt{5}}{2}$ & $\frac{1+\sqrt{5}}{2}$ & -1 & 1 & 1 & 1 \\
\hline 6 & 32 & 2 & 8 & $\mathbb{Q}(\sqrt{2})$ & $1+\sqrt{2}$ & $3+2 \sqrt{2}$ & +1 & 2 & 1 & 2 \\
\hline 11 & 117 & 3 & 13 & $\mathbb{Q}(\sqrt{13})$ & $\frac{3+\sqrt{13}}{2}$ & $\frac{11+3 \sqrt{13}}{2}$ & +1 & 2 & 1 & 2 \\
\hline 14 & 192 & 4 & 12 & $\mathbb{Q}(\sqrt{3})$ & $2+\sqrt{3}$ & $7+4 \sqrt{3}$ & +1 & 2 & 1 & 4 \\
\hline
\end{tabular}

Table 7 lists the conjugacy classes for $-15 \leq t \leq+15$. 


\begin{tabular}{|c|c|c|c|}
\hline$t=\operatorname{Tr} K$ & $\widehat{d}=t^{2}-4$ & $H(t)$ & $K$ \\
\hline 0 & -4 & 2 & $K=(0,1 ;-1,0), K^{-1}$ \\
\hline 1 & -3 & 2 & $K=(0,1 ;-1,1), K^{-1}$ \\
\hline 2 & 0 & $\infty$ & $K=(1,-n ; 0,1), n \in \mathbf{Z}$ \\
\hline 3 & 5 & 1 & $K=(0,1 ;-1,3)$ \\
\hline 4 & 12 & 2 & $K=(0,1 ;-1,4), K^{-1}$ \\
\hline 5 & 21 & 2 & $K=(0,1 ;-1,5), K^{-1}$ \\
\hline 6 & 32 & 2 & $K=(0,1 ;-1,6), K^{-1}$ \\
\hline 7 & 45 & 2 & $K=(0,1 ;-1,7), K^{-1}$ \\
\hline 8 & 60 & 4 & $\begin{array}{c}K_{1}=(0,1 ;-1,8) \\
K_{2}=(1,2 ; 3,7), K_{1}^{-1}, K_{2}^{-1}\end{array}$ \\
\hline 9 & 77 & 2 & $K=(0,1 ;-1,9), K^{-1}$ \\
\hline 10 & 96 & 4 & $\begin{array}{c}K_{1}=(0,1 ;-1,10) \\
K_{2}=(1,4 ; 2,9), K_{1}^{-1}, K_{2}^{-1}\end{array}$ \\
\hline 11 & 117 & 2 & $K=(0,1 ;-1,11), K^{-1}$ \\
\hline 12 & 140 & 4 & $\begin{array}{c}K_{1}=(0,1 ;-1,12) \\
K_{2}=(1,2 ; 5,11), K_{1}^{-1}, K_{2}^{-1}\end{array}$ \\
\hline 13 & 165 & 4 & $\begin{array}{c}K_{1}=(0,1 ;-1,13), \\
K_{2}=(2,3 ; 7,11), K_{1}^{-1}, K_{2}^{-1}\end{array}$ \\
\hline 14 & 192 & 4 & $\begin{array}{c}K_{1}=(0,1 ;-1,14), \\
K_{2}=(1,2 ; 6,13), K_{1}^{-1}, K_{2}^{-1}\end{array}$ \\
\hline 15 & 221 & 2 & $K=(0,1 ;-1,15), K^{-1}$ \\
\hline
\end{tabular}

Table 7: The number $H(t)$ of conjugacy classes of $S L(2, \mathbf{Z})$ at a given trace $t$, as well as the (factorized) discriminant $\widehat{d}$ of the associated quadratic form and matrix representatives for each class. In the last column the matrices are denoted as $(a, b ; c, d) \equiv\left(\begin{array}{ll}a & b \\ c & d\end{array}\right)$.

\section{References}

[1] C. Vafa, Evidence for F-Theory, Nucl. Phys., B 469 (1996), 403, hep-th/9602022. 
[2] M.R. Gaberdiel, B. Zwiebach, Exceptional groups from open strings, Nucl. Phys., B518 (1998), 151, hep-th/9709013.

[3] A. Johansen, A comment on BPS states in F-theory in 8 dimensions, Phys. Lett., B395 (1997), 36-41, hep-th/9608186.

[4] M.R. Gaberdiel, T. Hauer, B. Zwiebach, Open string-string junction transitions, Nucl. Phys., B525 (1998), 117, hep-th/9801205.

[5] O. DeWolfe and B. Zwiebach, String junctions for arbitrary Lie algebra representations, hep-th/9804210, to appear in Nucl. Phys., B.

[6] O. DeWolfe, T. Hauer, A. Iqbal, and B. Zwiebach, Uncovering the Symmetries of $[p, q]$ 7-branes: Beyond the Kodaira Classification, hep-th/9812028.

[7] O. DeWolfe, Affine Lie Algebras, String Junctions And 7-Branes, hep-th/9809026.

[8] O. DeWolfe, T. Hauer, A. Iqbal, and B. Zwiebach, Constraints On The BPS Spectrum Of N=2, $D=4$ Theories With $A-D-E$ Flavor Symmetry, hep-th/9805220, to appear in Nucl. Phys., B.

[9] Y. Imamura, E8 Flavor Multiplets, Phys.Rev., D58 (1998), 106005, hep-th/9802189.

[10] R.E. Borcherds, Vertex Algebras, Kac-Moody algebras, and the Monster, Proc. Nat. Acad. Soc. USA, 83 (1986), 3068;

R. E. Borcherds, Generalized Kac-Moody Algebras, Jour. Alg., 115 (1988), 501.

[11] V.G. Kac, R.V. Moody, and M. Wakimoto, On $E_{10}$, In K. Bleuer and M. Werner, editors, 'Differential geometrical methods in theoretical physics', Proceedings, NATO advanced research workshop, 16th international conference, Como, 1988.

[12] R.W. Gebert and H. Nicolai, On $E_{10}$ and the DDF Construction, Commun. Math. Phys., 172 (1995), 571, hep-th/ 9406175;

R.W. Gebert and H. Nicolai, $E_{10}$ for Beginners, in 'Gursey String \& Symmetries', (1994), 197, hep-th/9411188.

[13] J.A. Harvey and G. Moore, Algebras, BPS States and Strings, Nucl. Phys., B463 (1996), 315, hep-th/9510182. 
[14] J.A. Harvey and G. Moore, On the Algebras of BPS States, Commun. Math. Phys., 197 (1998), 489, hep-th/9609017.

[15] R. Dijkgraaf, The Mathematics Of Five-Branes, hep-th/9810157.

[16] V.A. Gritsenko and V.V. Nikulin, K3 Surfaces, Lorentzian KacMoody Algebras, and Mirror Symmetry, alg-geom/9510008.

[17] G. Moore, Arithmetic and attractors, hep-th/9807087.

[18] V. Kac, Infinite Dimensional Lie Algebras, Cam. Univ. Press, New York, 1985.

[19] W. Lerche and S. Stieberger, Prepotential, mirror map and Ftheory on $K 3$, hep-th/9804176.

[20] W. Lerche, S. Stieberger, and N.P. Warner, Quartic Gauge Coupling from K3 Geometry, hep-th/9811228.

[21] A. Klemm, P. Mayr, C. Vafa, BPS States of Exceptional NonCritical Strings, hep-th/9607139.

[22] M.R. Douglas, S. Katz, C. Vafa, Small Instantons, del Pezzo Surfaces and Type I' theory, Nucl. Phys., B497 (1997), 155-172, hep-th/9609071.

[23] J.A. Minahan, D. Nemeschansky, N.P. Warner, Investigating the $B P S$ Spectrum of Non-Critical $E_{n}$ Strings, Nucl. Phys., B508 (997), 64-106, hep-th/9707149.

[24] A. Kehagias, New type IIB vacua and their F-theory interpretation, Phys. Lett., B435 (1998), 337-342, hep-th/9805131.

[25] R. Donagi, A. Grassi, E. Witten, A non-perturbative superpotential with $E_{8}$ symmetry, Mod. Phys. Lett., A11 (1996), 2199-2212, hep-th/9607091.

[26] J. Minahan, D. Nemeschansky, C. Vafa, and N. Warner, Strings And N=4 Topological Yang-Mills Theories, Nucl. Phys., B527 (1998), 581, hep-th/9802168.

[27] O.J. Ganor, A test of $E_{8}$ chiral current algebra, Nucl. Phys., B479 (1996), 197-217, hep-th/9607020. 
[28] P. Aspinwall, K3 surfaces and string duality, hep-th/9611137.

[29] A. Sen, F-theory and Orientifolds, Nucl. Phys., B475 (1996), 562578 , hep-th/9605150.

[30] J.G. Wolfson, Minimal surfaces in Kähler surfaces and Ricci curvature, J. Differential Geometry, 29 (1989), 281.

[31] M. Bershadsky, V. Sadov, C. Vafa, D-branes and topological field theory, Nucl. Phys., B463 (1996), 420, hep-th/9511222.

[32] O. Ganor, D. Morrison, and N. Seiberg, Branes, CalabiYau Spaces, And Toroidal Compactification Of The $N=1$ Six-Dimensional $E_{8}$ Theory, Nucl. Phys., B487 (1997), 93, hep-th/9610251.

[33] B. Greene, A. Shapere, C. Vafa, and S. Yau, Stringy Cosmic Strings And Non-Compact Calabi-Yau Manifolds, Nucl. Phys., B337 (1990), 1.

[34] D.A. Buell, Binary quadratic forms, Springer-Verlag, New York, 1989.

[35] E. Hecke, Lectures on the theory of algebraic numbers, SpringerVerlag, New York, 1981.

[36] Z. Borevich and I. Shafarevich Number Theory, Academic Press, 1966.

[37] H. Cohn, A classical invitation to algebraic numbers and the class fields, Springer-Verlag, New York, 1978. 